\title{
Numerical and experimental examination of turbulent mixing of a heated jet in crossflow
}

\author{
Michael R. Borghi Jr.* Douglas Thurman ${ }^{\dagger \dagger}$ Philip Poinsatte ${ }^{\ddagger \ddagger}$ \\ NASA Glenn Research Center, Cleveland, OH., 44135 \\ William A. Engblom ${ }^{\dagger \S}$ \\ Embry-Riddle Aeronautical University, Daytona Beach, Fl., 32114
}

\begin{abstract}
The injection of fully-developed turbulent heated air from a tube into a cooler turbulent duct flow is examined, as an analogy to film cooled turbine blades. A LES numerical model is developed and applied in which tube and duct turbulence inflow effects are emulated using a divergence-free synthetic eddy method (SEM). For direct comparison, a hot-wire experiment is conducted within the ERB test cell SW-6 at NASA Glenn Research Center. Results related to velocity, temperature, and heat flux are obtained numerically and experimentally for a blowing ratio of 1.2 , involving a $36 \mathrm{~K}$ temperature difference. The relative effect on the solutions of tube and duct inflow turbulence is systematically evaluated. The impact of inherent low-pass filtering of temperature measurements and probe wire offset on the experimental results are addressed.
\end{abstract}

\section{Nomenclature}

Blowing ratio

Temperature ratio

Turbulent dissipation rate

Specific rate of dissipation,

Turbulent kinetic energy

Cooling hole diameter

Boundary layer thickness

Distance away from nearest wall
$T \quad$ Temperature
$T^{\prime} \quad$ Temperature fluctuation
$\mathrm{Pr}_{t} \quad$ Turbulent Prandtl number
$X, Y, Z$ Cartesian spatial components
of streamwise, vertical, spanwise
$U, V, W$ Cartesian velocity components

\section{Introduction}

Film cooling has remained an active area of research over the previous five decades. ${ }^{1-3}$ It is a key part of the thermal management of various propulsion system components such as turbine blades and afterburners. The push for improved performance and efficiency will continue to drive hot-section temperatures higher. Even with advanced materials, such as ceramic matrix composites, ${ }^{4}$ and thermal barriers/coatings, there remains a need for active cooling. The validation of analytical turbulent heat flux models for design purposes ${ }^{5,6}$ are limited by a lack of high quality experimental and numerical data for complex flow fields. Accurate and efficient numerical techniques, validated by detailed experiments, are sought to support advancement of turbine film cooling technologies.

\footnotetext{
*Aerospace Engineer, Turbomachinery and Turboelectric Propulsion Branch, Member AIAA.

${ }^{\dagger}$ Aerospace Engineer, Turbomachinery and Turboelectric Propulsion Branch, Army Research Lab

¥Aerospace Engineer, Turbomachinery and Turboelectric Propulsion Branch

$\S$ Professor, Departments of Mechanical and Aerospace Engineering, Associate Fellow AIAA.
} 


\section{A. Film Cooling Physics}

Film cooling applications are a small subset of a larger set of flow phenomena: jets in crossflows. A key parameter in classifying the jet in crossflow problem is the blowing ratio,

$$
B R=\frac{\rho_{j} U_{j}}{\rho_{\infty} U_{\infty}}
$$

where $\rho_{j}$ and $U_{j}$ are the density and velocity of the jet respectively, and $\rho_{\infty}$ and $U_{\infty}$ are the properties of the crossflow. The forces driving the flow are of a different nature for low blowing ratios $(B R<2)$ versus higher blowing ratios. ${ }^{7}$ For higher blowing ratios, the region near the jet is driven by inviscid fluid dynamics, with turbulence effects not playing a role until further downstream. Lower blowing ratios, however, have near fields which are dominated by turbulence effects, and have the added complication of largely non-uniform velocity profiles near the jet exit. Figure 1(a) shows schematic depictions of the difference in blowing ratios.

As shown in Fig. 1(a) as $B R$ increases the more the jet is lifted off the surface. This lifting causes flow from either side of the jet to move towards the plane of symmetry. This flow then recirculates and moves upstream towards the jet and ultimately entrained into the jet. This increased recirculation region in higher blowing ratio flows leads to low film cooling efficiency, and thus is less suitable for film cooling applications.

Fric and Roshko ${ }^{8}$ identify four main regions within the near-field region of the jet in crossflow. These regions are:

- Jet shear-layer vortices - these vortices have their origin within the boundary layer within the jet, and grow due to a Kelvin-Helmholtz instability within the jet-freestream shear-layer. ${ }^{8}$

- System of horseshoe vortices - these vortices are the result of an adverse pressure gradient just upstream of the jet, along with vorticity which exists within the boundary layer upstream of the jet. ${ }^{9}$

- Counter-rotating vortex pair (CRVP) - these dominate the flow downstream of the injection site. According to Cortelezzi \& Karagozian these are a result of a process of rolling up, tilting, and folding over shear layer vortices. ${ }^{10}$

- Wake vortices - the least understood of the near field phenomena, are likely generated from the boundary layer downstream of the jet. These vortices will play a large role in the effectiveness of film cooling applications of a jet in crossflow. ${ }^{8}$

Of these four regions, two are inherently unsteady phenomenon: the jet shear-layer and wake vortices. Modeling these are beyond the capability of conventional RANS methods. Capturing their effects will therefore require an eddy-resolving time accurate method. The other regions of the flow, while possibly containing unsteady components, have strong base flow representation and may more adequately be captured by steady flow methods. Figure 1(b) shows a schematic depiction of the four dominate regions of the near field region of a jet in crossflow.

\section{B. Numerical Background}

The use of Reynolds averaged Navier-Stokes (RANS) turbulence closures presents limitations for aerothermal systems analysis. Conventional turbulence modeling assumes a dynamic similarity between heat and momentum transfer, via usage of a constant turbulent Prandtl number $\operatorname{Pr}_{t}$. Variable $\operatorname{Pr}_{t}$ models have had difficulty matching experimental data. ${ }^{11-14}$

Scale resolving simulations (SRS) have become a major focus in recent years. Detached Eddy Simulation (DES) and Large Eddy Simulation (LES) allow the largest scales of turbulence to be resolved while filtering (implicitly or explicitly) the smallest scales. Recent work by the authors has shown that the use of Detached Eddy Simulations (DES) does not provide sufficiently accurate results for a heated jet in a crossflow. Without a mechanism to convert modeled turbulence ( $\mu_{t}$ via standard RANS models) into unsteady, physical, "large scale" turbulent structures, a significant delay in the mixing process was observed relative to experimental data. ${ }^{15}$ This delay led to under predicted stresses in the near field and over predicted stresses downstream. Other researchers employing LES have investigated various aspects of film cooling with varying levels of success. Johnson et al. showed good results using a recycling and rescaling inflow turbulence method. Instead of solving the energy equation, temperature was treated as a passive scalar. ${ }^{16}$ The effects of density 
ratio on the rms velocity and temperature fields have been studied by Renze et al. though the use of a $\mathrm{CO}_{2}$ coolant. ${ }^{17}$

In LES cases, the generation of the inlet flow conditions is an important aspect of the simulation. In many cases, without a forced disturbance, a steady inflow condition will remain in a stable laminar condition and never fully transition to turbulent flow, if at all. A long standing practice has been to use a precursor simulation to generate the inflow boundary data via a procedure such as recycling and rescaling. ${ }^{18}$ This approach however requires a relatively simple geometry and significant extra computational expense and time. These limitations have led to the development of synthetic turbulence generation methods. Initially, random noise was applied to a mean flow as a method of perturbing the freestream flow. The lack of coherent structures in time and space lead to a rapid decay of the turbulent energy. ${ }^{19}$ Klein et al. ${ }^{20}$ developed a methodology to digitally filter random data on to the inlet mesh. Jarrin et al. devleoped the synthetic eddy method (SEM), which takes a mean velocity profile and Reynolds stress tensor to create coherent turbulent structures at the inflow durring the runtime of the simulation. ${ }^{21,22}$ Recently, developments have been made to the SEM approach to improve the accuracy and efficiency as well as the ability to produce a divergencefree turbulent flow field. ${ }^{23-25}$ For the present study, the divergence-free synethetic eddy method is selected for use in the LES simulations.

SRS's high computational cost prevents them from fully replacing RANS-based turbulence models commonly used for design purposes. However, the insight gained into the fundamental flow physics by the use of highly resolved simulations can help reshape conventional RANS models. Muldoon et al. analysised $k-\epsilon$ budgets with the results of a DNS simuluation. The jet exit in Muldoon et al. was modeled with a velocity boundary condition. ${ }^{26}$ They can also be the basis for other simplified momentum source term based models for even less computational expense. ${ }^{27}$ This is a primary long-term motiviation for developing the validated, high-fidelity LES simulations presented in this work.

\section{Experimental Background}

A major limitation on validating numerical models is the limited number of high quality experimental data sets containing the relevant heat transfer related turbulence quantities. In fact, some studies have reported aphysical trends in the turbulent quantities measured. ${ }^{28}$ Temperature fluctuations $\left(T^{\prime}\right)$ and turbulent heat fluxes $\left(\overline{u^{\prime} T^{\prime}}\right)$ are key parameters which are not commonly or easily measured. High frequency temperature measurements using fine-wire thermometers are difficult to take due to the low cut off frequencies operating in constant current mode (CCA). ${ }^{29-32}$ For heat flux measurements, simultaneous measurements of velocity and temperature must be taken. The use of constant temperature anemometry (CTA) or hotwires are often used to obtain velocity measurements. The physical separation of these measurements (i.e., distance between velocity and temperature sensors) can introduce significant error to the correlation. For the present study, multiple hot-wire probes and post-processing techniques are utilized in an effort to obtain a high-quality turbulence-related data set.

\section{Experiment Methodology}

\section{A. Experimental Setup}

An experiment was conducted in the wind tunnel at NASA Glenn Research Center, ERB test cell SW-6, to acquire turbulent heat flux measurements on a large scale model of film cooling/heating holes. The test facility, shown in Fig. 2, consists of an aluminum bellmouth, flow conditioning honeycomb and screens, and a square acrylic section $0.2 \mathrm{~m} \times 0.2 \mathrm{~m}$ wide with $1.90 \mathrm{~cm}$ thick walls. The tunnel was connected to a central exhaust system downstream that pulled room air through. The test section consisted of an interchangeable floor section fabricated from ABSplus thermoplastic in a 3-D printer, with three injection holes for periodic flow and boundary conditions. The hole diameter was $1.905 \mathrm{~cm}$ and was inclined at $30^{\circ}$ from the horizontal surface. The spanwise spacing was set to $Z / D=3$.

A PC-based data acquisition system was used to acquire data from pressure transducers and thermocouples. The tunnel flow rate was measured from a total pressure probe placed just upstream of the test section and static pressure taps located on the sidewalls at the same plane. Freestream temperature was measured with a thermocouple located upstream of the holes near the total pressure probe. Tunnel flow was nominally set to $9.14 \mathrm{~m} / \mathrm{s}$ at ambient conditions which corresponded to a Reynolds number based on hole diameter and freestream velocity of 11,000 . Freestream turbulence was measured to be less than $1 \%$ at the inlet to 
Table 1. Nominal Experimental Conditions

\begin{tabular}{c|ccccccc} 
Case & $V\left(\frac{m}{s e c}\right)$ & $\rho_{o}\left(\frac{\mathrm{kg}}{\mathrm{m}^{3}}\right)$ & $T_{o}(K)$ & $V\left(\frac{m}{\sec }\right)$ & $\rho_{o}\left(\frac{\mathrm{kg}}{\mathrm{m}^{3}}\right)$ & $T_{o}(K)$ & $\mathrm{BR}$ \\
& Tunnel & Tunnel & Tunnel & Tube & Tube & Tube & \\
\hline 1 & 9.14 & 1.174 & 296.5 & 13.7 & 1.038 & 336.0 & 1.2 \\
2 & 9.14 & 1.169 & 294.8 & 25.3 & 1.032 & 336.0 & 2.4
\end{tabular}

the test section, and boundary layer thickness measured around $2 \mathrm{~cm}$ upstream of the injection holes.

The injection flow was provided by blowing pressurized air through a flow meter and into a plenum attached to the underside of the test section floor plate. The tubes connecting the plenum to the test section holes had a length of approximately $L / D=20$ which is sufficient to ensure a turbulent velocity profile in the tube. The injection flow conditions were measured with static pressure taps and thermocouples both inside the plenum and in each injection tube. In previous film cooling tests with this tunnel, the injected air was cooled by passing through copper tubing that was coiled inside an ice water tank. ${ }^{33}$ However for this effort, a larger temperature difference was desired, so the injection air was heated using three $750 \mathrm{~W}$ in-line electrical pipe heaters. This provided a temperature difference of approximately $36{ }^{\circ} \mathrm{K}$. Note that this heated jet configuration would not be typical in a turbomachinery application, but is an appropriate analogy.

Three different probe configurations were used to take velocity and temperature measurements. A $3.8 \mu m$ single wire probe was initially used for temperature measurements. Two different 3 -wire probes ( $\mathrm{x}$-T probes) were then used to make simultaneous velocity and temperature measurements. Both sets of $\mathrm{x}-\mathrm{T}$ probes consisted of a $1.2 \mu \mathrm{m}$ wire $0.4 \mathrm{~mm}$ in length for temperature measurements(T-wire). Velocity $(\rho V)$ measurements were made using two $25 \mu \mathrm{m}$ wires (x-wires) positioned perpendicularly to one another. The law of cosines was then used to reconstruct to velocity components from the two perpendicular velocity signals. The T-wire was connected to a CCA system to measure temperature, while the $\mathrm{x}$-wires were operated in CTA mode to obtain velocity. One $\mathrm{x}-\mathrm{T}$ probe was oriented for $\mathrm{U}$ - and $\mathrm{V}$ - velocity measurements, while the other probe measured U- and $\mathrm{W}$ - velocity components. The U-V and U-W velocity measurements were taken independently of each other. The T-wire in the U-V probe was slightly offset in the spanwise (W velocity direction), while the U-W probes T-wire was vertically offset (V velocity direction). It is found that these wire offsets must be accounted for properly when evaluating turbulence quantities. The $\mathrm{x}$-T probes used here have probe volumes of roughly $0.15 D x 0.2 D x 0.15 D$ with the long dimension oriented in the secondary velocity direction (ie. in the $\mathrm{V}$ direction for the U-V probe).

Pressure, temperature, and velocity surveys were taken along the centerline in the streamwise direction and at cross sectional planes $X / D=2,3,4,6$, and 10, as measured from the hole leading edge. An actuator system was used to position the probe in the tunnel. The pressure surveys were taken with a pitotstatic probe. Data at nominal blowing ratios of 1.2 and 2.4 were acquired. Both velocity and temperature measurements were averaged for 3 seconds at a frequency of $50 \mathrm{kHz}$. The surveys were completed over several days.

The probes were calibrated in a separate calibration tunnel by varying the velocity and temperature of the flow and recording the voltages for each wire. Since the CTA hot-wire is sensitive to the temperature of the flow, a series of calibration curves at various velocities and temperatures were used to interpolate $\rho V$ from the wire voltage. Static pressure and temperature surveys were used to calculate a mean density to obtain the velocity. Table 1 lists the conditions considered for the experimental data collected. However, numerical simulations were only conducted at $B R=1.2$, therefore only the lower blowing ratio will be discussed herein. The higher blowing ratio will be the topic of future paper.

\section{B. CCA frequency compensation}

To account for the low-pass filtering inherent to CCA measurements, the numerical compensation technique of Tagawa et al. ${ }^{29}$ was implemented. The technique provides a closed form equation for the frequency response of a given fine-wire thermometer. The model is based solely on physical properties of the probe, and the local flow properties. It can therefore be easily applied to a wide range of CCA probes without the need for extra characterization experiments.

The goal of the technique is to provide a transfer function modeling the frequency response of the CCA probe. The approach treats the individual components of the CCA probe separately, linked together though 
boundary conditions. The final transfer function is given by,

$$
H(\omega)=\frac{1}{1+j \omega \tau_{1}}-\frac{\left[\frac{1}{\left(1+j \omega \tau_{2}\right)}-\frac{1}{\left(1+j \omega \tau_{3}\right)}+\left(\frac{1}{1+j \omega \tau_{1}}-\frac{1}{1+j \omega \tau_{2}}\right) \cosh \left(\frac{\Omega(L-l)}{2}\right)\right]}{\left[\frac{\Omega_{1} l}{2}\left(\cosh \left(\frac{\Omega_{1} L}{2}\right) \cosh \left(\frac{\Omega_{2}(L-l)}{2}\right)\right)+\frac{\Omega_{1} \lambda_{1} d_{1}^{2}}{\Omega_{2} \lambda_{2} d_{2}^{2}} \sinh \left(\frac{\Omega_{2}(L-l)}{2}\right)\right]}
$$

The time constant and a wire parameter are given by,

$$
\begin{aligned}
\tau_{i}=\frac{\rho_{i} c_{i} d_{i}^{2}}{4 N u \lambda_{g}}, & i=1,2,3 \\
\Omega_{i}=\sqrt{1+\frac{j \omega \tau_{i}}{a_{i} \tau_{i}}}, & i=1,2
\end{aligned}
$$

Above, the subscripts represent the different components of the CCA probe. The first, $i=1$ is the sensor itself. Next, is the support stubs $i=2$. The prongs are represented by $i=3$. Here, $\rho_{i}, c_{i}, \lambda_{i}, a_{i}$ is the density, specific heat, thermal conductivity, and thermal diffusivity respectively of the $i^{\text {th }}$ CCA component. The Nusselt number, $\mathrm{Nu}$, can be evaluated using empirical relations for flow around a cylinder. The nondimentional time constant for the prongs, $\tau_{3}$ is assumed to be unity. The transfer function must be applied to the temperature signal in the frequency domain. The Fast Fourier Transform (FFT) of the time series temperature data is computed, and the compensation transfer function applied,

$$
\begin{aligned}
Y(\omega) & =F F T(T(t)) \\
Y_{c}(\omega) & =\frac{Y(\omega)}{H(\omega)}
\end{aligned}
$$

The compensated temperature spectrum is then returned to the time domain through the application of an IFFT,

$$
T_{c}(t)=\operatorname{IFFT}\left(Y_{c}(\omega)\right)
$$

$T_{c}(t)$ is the compensated temperature signal as a function of time. The new estimated temperature turbulent statistics may be computed. For the derivation and more information of the compensation approach applied here see the orginal paper of Tagaw et al. ${ }^{29}$

\section{Numerical Model and Methodology}

\section{A. Numerical Scheme}

The OpenFOAM software package is used to perform large eddy simulation (LES) on the jet in crossflow cases examined herein. The pressure-based rhoPimpleFOAM solver is utilized to solve the Navier-Stokes and energy equations. This solver combines a PISO-like time marching scheme with the pressure-velocity coupling of the SIMPLE algorithm. The energy equation is formulated with the calorically-perfect, sensible internal energy, and the ideal gas law is used for the equation of state. Sutherland's law is used for the gas viscosity and thermal conductivity. Bounded second order schemes are used for the spatial derivatives, and a Crank-Nicolson scheme is used for time advancement. The Crank-Nicolson scheme includes an off-centering coefficient which can be used to increase stability at the cost of overall scheme order. A coefficient of 0.25 was required for stability across all of the simulations. Stability was most limiting with SEM applied within the tube.

\section{B. Grid and Boundary Conditions}

After an unreported initial coarse grid simulation, a fine grid was used for the simulations within this paper. The grid was packed to the channel walls, with the first point well within the viscous sublayer, with a normal-to-wall spacing set to $25.4 \mu \mathrm{m}$ to obtain a target of $y^{+} \leq 1$, based on a tunnel velocity of $9.14 \mathrm{~m} / \mathrm{s}$. The side walls are periodic to simulate a infinite span of injectors. The injector topology was comprised of 5 structured zones in an $\mathrm{O}-\mathrm{H}$ formation. These zones then extend vertically through the tunnel domain.

The grid has an extended tunnel inflow (+7D) to allow for SEM transition bring the domain to $X \in$ $[-10.33 D, 14 D]$ with $\mathrm{Y}$ and $\mathrm{Z}$ extents remaining the same. In the fine grid the spanwise spacing is mostly 
constant and capped at $\Delta Z^{+} \approx 9$, but is reduced in regions near the hole exit. The streamwise spacing is capped at $\Delta X^{+}=20$, the grid is allowed to stretch slightly far downstream of the hole $\left(\frac{X}{D} \approx 12\right)$. The wall normal spacing is significantly refined stretching to a maximum of $\Delta Y^{+}=25,2 \mathrm{D}$ above the tunnel floor. The grid is comprised of roughly 68 million cells. Figure 3 shows the extent of the computational domain used in the computations.

The boundary conditions are identical for each grid,

- Tunnel Inflow: A fully developed velocity profile and Reynolds stress profile (for SEM) are applied. The fine grid inflow is split into two regions to limit the extent of the SEM to the boundary layer only. The profiles are obtained from a $k-\omega$ SST solution.

- Outflow: Static pressure is imposed at the outflow domain, and the velocity is held to a zero-gradient normal to the exit.

- Cooling Holes Inflow: Similar to the tunnel inflow, a fully developed velocity profile and Reynolds stress profile (for SEM) is applied. The profiles are obtained from a $k-\omega$ SST solution.

- Viscous Walls: Walls are treated as viscous and adiabatic.

- Cyclic: The solution is periodic in the spanwise direction.

- Freestream: The freestream boundary (i.e. parallel to the tunnel floor) is treated with an inlet-outlet type condition. The inlet-outlet boundary acts a velocity inlet and pressure outlet based on the local velocity vector.

A flat plate grid, was also used for the validation cases of the SEM. This grid is identical to the SEM inflow region of the film cooling grid described above. The grid was approximately $[10 \delta x 6 \delta x 3 \delta]$ in the $\mathrm{x}, \mathrm{y}$, and $\mathrm{z}$ directions respectively. The grid spacing was fixed to $\Delta X^{+}=15$ and $\Delta Z^{+}=8$ in the stream- and spanwise directions. Wall normal spacing was $\Delta Y^{+} \leq 1$ at the wall and allowed to stretch to no more than $\Delta Y^{+}=25$ at the edge of the boundary layer. Roughly 14.5 million cells are used for the the entire domain. The inlet and outlet boundary conditions match those used in the film cooling simulations.

\section{Numerical Procedure}

The unsteady LES simulations are initialized in an efficient manner. First, the entire domain is computed using a steady RANS approach. Then, the tube and duct transition regions (i.e., prior to the crossflow region) are each independently computed using unsteady LES with the divergence-free SEM applied at the inflow boundaries (with steady RANS solution used to initialize the LES). For cases in which SEM is not applied to either the tube or duct inflow boudnary, the velocity profile from the steady RANS solution is applied. Once a proper start-up time period has been computed, these intermediate tube and duct transition solutions are mapped back onto the full tube-duct domain, and the steady RANS solution remains intact elsewhere in the domain. It should be noted that to maintain a consistent boundary layer and momentum thickness at the inflow boundaries for cases in which SEM is turned off at the tube or duct inflow boundary, it was necessary to replace the RANS-based inlet velocity profiles with time-averaged inlet velocity profiles generated by the full SEM case (i.e., the baseline case in which SEM is applied to both tube and duct).

Substantial computational effort is required to obtain final turbulence statistics. The flow through time from the jet exit to domain exit is roughly $\tau=0.01667$ seconds. The solution is computed for $6 \tau$ before averaging and time-statistics start to be collected. This allows the solution to reach a quasi steady state, and for transient interactions between the tunnel and jet flows to be propagated and dissipated through the domain. Time statistics and averaging is then conducted over another $12 \tau$. This provided sufficient time for statistical quantities to converge. A timestep of $1 \times 10^{-6}$ seconds $\left(\Delta \tau=5 \times 10^{-5}\right)$ is used for both the initial FTTs and the averaging period.

\section{Results}

\section{A. Validation of Numerical Model}

A validation of the SEM was performed on a flat plate case with a similar Reynolds number based on momentum thickness $\left(R e_{\Theta}\right)$ to the film cooling case of interest. A 2D RANS simulation $(k-\omega \mathrm{SST})$ was used 
to obtain inputs for the SEM boundary. Mean velocity and isotropic Reynolds stress profiles at $R e_{\theta}=1280$ and a freestream velocity of $9.14 \mathrm{~m} / \mathrm{s}$ were mapped to the SEM inlet boundary. Figure 9(a) shows the span averaged friction coefficient develop downstream. Figure 9(b) shows Q-criterion colored by the normalized axial Reynolds stress. The initial freestream turbulence provided from the RANS profile quickly dissipates within the first three boundary layer thicknesses downstream of the inlet. The small structures associated with the inlet synthetic turbulence start to form larger structures roughly half way through the domain. Figure 9 (b) shows the axial stress profile initially growing before normalizing down again around $\frac{X}{\delta}=6$. This is due to the transition process required to transform the synthetic unnatural turbulence at the inflow to the realistic structures seen downstream. This transition process is sensitive to the number and size of eddies used to poulate the the inflow domain, but the friction coefficient begins to plateau close to the expected DNS-derived result of $0.0034 .^{34}$

In Fig. 10 Reynolds stress profile at various downstream locations of the SEM inlet are compared to the corresponding profiles form DNS. The local momentum thickness Reynolds number is provided for reference at each location. The axial stress is reasonably well represented at the $\frac{X}{\delta}=8.0$ location. The first two stations $\left(\frac{X}{\delta}=2.5,5.3\right)$ however, significantly under predict the axial stress. This is related to the transition process described above. All locations under predict the spanwise and wall-normal stresses, $\overline{w^{\prime} w^{\prime}}$ and $\overline{v^{\prime} v^{\prime}}$ respectively. The shear stress remains roughly constant across the range of Reynolds numbers seen here. Across all of the stresses, the outer wake region $\left(y^{+} \geq 150\right)$ is thinner than the DNS data suggests. Moderate grid stretching could be a factor in the wake region discrepancy. Based on these acceptable results, this SEM flat plate case was also used to tune SEM model settings, set the required grid fineness for SEM transition, and obtain an initial duct transition solution for the film cooling simulations. A similar study was performed for the turbulent flow in the tube.

\section{B. xT-Probe Performance Assessment}

The effect of the cut-off frequency on probe measurement accuracy is evaluated for both the $1.2 \mu m$ and $3.8 \mu \mathrm{m}$ diameter hot-wires. Using the method of Tagawa ${ }^{29}$ described above, the cutoff frequency for both thermometers are estimated analytically. Figure 4 shows the cutoff frequency as a function of the local velocity and temperature. The $3.8 \mu \mathrm{m}$ wire is seen to have a cutoff frequency roughly an order of magnitude lower than that of the $1.2 \mu \mathrm{m}$ wire. The kink in the curves is due to a change in empirical Nusselt number relations to account for Reynolds numbers less than 2. Centerline surveys of temperature are shown in Fig. 5 with and without numerical compensation. The uncompensated temperature variance $\left(\frac{T_{r m s}}{\Delta T}\right)$ shows significant difference between the two wire diameters. The low cutoff frequency does not just affect the spectral content of the measurement, the thermal inertia effects cause peak temperatures to be under represented, and therefore the variance of the measurements. However, once the compensation is added for the $3.8 \mu \mathrm{m}$ wire, the thermal variance closely matches that from the uncompensated $1.2 \mu \mathrm{m}$ wire measurement. Note that the compensation methodology can only account for a finite level of low pass filtering, typically around one order of magnitude. Consequently, for the remainder of the results section, only $1.2 \mu \mathrm{m}$ compensated temperature results will be shown.

The sensitivity of temperature measurements to the orientation of the xT-probes (i.e., the UV and UW probes) is also evaluated. Fig. 6 shows good agreement between the two probes (UV and UW) for both mean normalized temperature and normalized temperature variance. Here the vertical shift in the UW- probe has been accounted for during post-processing. It should be noted that centerline and spanwise measurements are typically taken on different days, and so, the variation between centerline and spanwise measurements is possibly attributed to day-to-day experimental variations.

The sensitivity of streamwise velocity between the two xT-probes is also presented in Fig. 7. The measured freestream normalization velocity, $U_{\infty}$, has been increased to account for the slightly higher freestream velocities recorded with the UW-probe, while $U_{\infty}$ remains unchanged for the UV probe. The streamwise velocity does not exhibit the same good agreement between probes as the temperature. The mean jet Uvelocity is roughly $13 \%$ higher when measured by the UW-probe. There is also a significant difference in the spanwise mean velocity profiles. At $X / D=2.00$ a significant bias is to the positive $Z / D$ direction is seen with the UV-probe, whereas the UW-probes exhibits a more symmetric profile. This asymmetry is unlikely to be attributed to the flow field, as both temperature measurement sets shows very good symmetry.

The aforementioned streawise velocity discrepancies likely result from bias caused by non-resolvable velocity components influencing the two $\mathrm{x}$-wires unevenly. The UV-probe expects no spanwise velocity, whereas the UW-probe assumes no vertical velocity. Using the flow field results from the Fulll-SEM LES 
simulation, the local flow angles were computed and are shown in Fig. 8 to support this argument. Along the centerline most of the flow has a relative flow angle upwards of $20^{\circ}$, far from ideal for the UW-probe. Off-center the spanwise flow angle of found to be in the $\pm 15^{\circ}$ range in the regions of highest bias for the UV-probe. These unwanted velocity components, and related flow angle changes, appear to greatly increase the uncertainty of the probe measurements in these regions.

\section{Comparison of LES with Experimental Data}

The effect of physical, resolved turbulence in LES simulations of the film cooling problem was studied with a test matrix of cases. The cases were composed of: Full-SEM, Tube-SEM, and Tunnel-SEM. The Full-SEM case had synthetic turbulence generated at both the tunnel and tube inflow boundaries, while the Tube-SEM and Tunnel-SEM only generated inflow turbulence at the tube and tunnel boundaries respectively.

Figure 11 compares the Full-SEM with the experimental data taken with the UV-probe. Excellent agreement is seen between the Full-SEM and hot-wire for mean temperature measurements (upper left image), with overall thickness and gradients being well represented. Good agreement is also obtained with thermal standard deviation (lower left image), with peak values slightly over predicted by the LES. Temperature measurements made at the trailing edge of the jet exit $(\mathrm{X} / \mathrm{D}=2.00)$ suggest a modest thermal boundary layer exits within the jet, based on the small reduction of mean temperature and the presence of a small thermal variance near the wall $(\mathrm{Y} / \mathrm{D}=0.00)$. This suggests the adiabatic walls used in the LES are not strictly correct. However, the nonadiabaticity of the tube has little impact elsewhere in the flow domain. The over prediction could be exaggerated slightly by the lower resolution of the CCA data not fully resolving peaks in the profile close to the wall.

Figure 11 also shows reasonable agreement is obtained for mean and root-mean-squared streamline velocity (see middle images). The peak streamwise velocity at the hole exit is elevated slightly compared to the CTA measurements. Streamwise velocity fluctuations agree well at the jet exit as well as downstream at $\mathrm{X} / \mathrm{D}=6.00$. In the mid region $(\mathrm{X} / \mathrm{D}=4.00)$, the magnitude matches well; however, LES results show a series of peaks which are not resolved by the CTA data. Since the X-wires of the UV-probe are oriented in the vertical direction, this orientation stretches the probe volume vertically and can cause smaller flow features to be smeared. This is especially true when gradients are strong in the stretched direction, as is the case here. Finer measurement resolution as well as post processing techniques could mitigate this resolution issue. The mean vertical velocity profiles show good qualitative agreement between CTA data and LES, with LES peak velocities slightly elevated near the jet exit.

Fig. 12 depicts the normalized mean temperature contours for the SEM sensitivity cases as well as UVprobe CCA data (lower right image). The differences between the three SEM treatment cases are subtle. The full-SEM simulation shows the highest film effectiveness with the $\Theta=0.2$ contour reaching the wall just after the $\mathrm{X} / \mathrm{D}=4.00$ location. The tube-SEM and tunnel-SEM cases also show reattachment with the $\Theta=0.2$ contour reaching the wall at around 6 and 7 diameters downstream, respectively. The tunnel-SEM case also exhibits a a slightly more noticeable cold-spot after the jet exit. The shear layer growth of both the full- and tube-SEM cases show excellent agreement, while the tunnel-SEM shear layer's growth is delayed until $\mathrm{X} / \mathrm{D}=1.00$.

Fig. 13 depicts normalized temperature variance contours. The full- and tube-SEM simulations are very similar with only subtle differences, such as the slightly longer extent of the underside portion of the wake. The distribution of thermal variance in the tunnel-SEM configuration is significantly different from the other SEM cases. Due to the reduced shear layer growth temperature fluctuations of up to $40 \%$ are seen in the shear layer until X/D $=3.00$. Additionally, both the extent and magnitude of the underside wake is increased in comparison to the other SEM cases. The circumferential extent of $T_{r m s} / \Delta T$ and radial gradients are also larger in the tunnel-SEM simulation. Without the turbulence present within the tube to enhance eddy transport approcing the mixing region, the resulting mixing of the hot and cold gases is less vigorous, leading to higher temperature gradients and variance.

Figures 14 and 15 contain mean streamwise and vertical velocity contours, respectively. Again, the full- and tube-SEM simulations share many similarities with subtle differences. The jet exit velocity for the tunnel-SEM case is elevated compared to the simulations which include tube turbulence. The applied velocity boundary condition profile in the tunnel-SEM is a time-average of the full-SEM tube 3 diameters upstream of the hole exit. The presence of turbulence within the tube causes the velocity profile to widen and become flatter, this leads to the lower peak velocities in the full- and tube-SEM cases. Similarly, vertical velocity profiles for the tunnel-SEM penetrate further into the crossflow and exhibit a larger secondary up-wash. 
Fig. 16 illustrates the root-mean-squared streamwise velocity contours. In the LES simulations, a region of strong streamwise velocity fluctuation extends from the trailing edge of the hole to the end of the jet penetration $(\mathrm{X} / \mathrm{D} \approx 4.5)$. However. the hot wire data contains a region of less strong fluctuations which appears to stem more from the upper shear layer and the hole leading edge. The larger probe height $(\approx 0.25 D)$ and fairly coarse probe resolution could lead to smaller flow features, like the strong fluctuations noted in the LES, to become vertically smeared. It should be noted that the vertical thickness of the underside shear layer is typically smaller than the vertical probe length. At $\mathrm{X} / \mathrm{D}=4.00$ the peak $U_{r m s} / U_{\infty}$ region, from the full-SEM simulation, extends a vertical distance of roughly $0.12 \mathrm{D}$, or half of the probe length.

Fig. 17 shows the most significant difference amongst the three SEM cases. While the full- and tubeSEM simulations again show only minor differences the tunnel-SEM simulation contains significantly more vertical fluctuation at the end of the jet penetration $(\mathrm{X} / \mathrm{D} \approx 4.5)$. This region roughly correlates with the secondary up-wash seen in the mean vertical velocity. The presence of tube turbulence significantly breaks down the strong coherent K-H type structures into smaller more three-dimensional structures, redistributing the turbulent energy elsewhere. The UV probe shows slightly weaker peak $V_{r m s} / U_{\infty}$ magnitudes and the extent of the peak region is slightly more limited. The probe volume and resolution could impact the results here; however the larger size of the feature of interest suggests this may not be the case. The local flow angle, as shown in Fig. 8(a), suggests that the local flow conditions are on the edge of the acceptable flow angles for the UV probe. Spanwise velocity fluctuations are plotted in Fig. 18. Similar trends are seen here as with the other velocity and temperature fluctuations. The initial shear layer growth is limited in the tunnel-SEM simulations compared to the other LES simulations, and a slightly more energetic underside wake region is produced.

Figure 19 is related to the streamwise turbulent heat flux. It contains the product of $U_{r m s}$ and $T_{r m s}$, which gives an estimate of the heat flux without the effect of signal correlation. As root-mean-squared velocity and temperature contours are both in good agreement with experimental profiles it follows that the uncorrelated heat flux should also be in good agreement. The primary disagreement between LES and experimental comes from the underside shear layer region, where the LES contains elevated $U_{r m s}$ profiles. This is a carry-over effect from the $U_{r m s}$ profiles.

Figure 20 contains the vertical turbulent heat flux, $\overline{V^{\prime} T^{\prime}} / U_{\infty} \Delta T$. However, the LES results do not account for the fact that the velocity and temperature probes are separated by a significant distance, on the order of $\frac{D}{10}$, much larger than the average turbulent length scale. This loss of signal correlation has a dramatic effect since the LES results no longer agree well with the experimental profiles either quantitatively or qualitatively. The use of the numerical compensation techniques described earlier also limits the validity of the heat flux measurements since the reconstructed signal is not influenced by the local velocity spectrum and therefore biases the correlation. A similar analysis has been done for the streamwise heat flux, $\overline{U^{\prime} T^{\prime}} / U_{\infty} \Delta T$. The same issues for the vertical component of the heat flux affect the streamwise direction. Future work should be done to apply the two-point correlation representative of the experimental measurement to the LES solutions.

\section{Conclusions}

An experimental and numerical investigation into turbulent mixing of a heated jet in a crossflow was conducted at NASA Glenn Research Center, ERB test cell SW-6, and via a set of high-fidelity LES simulations with inflow turbulence driven by a divergence-free SEM model.

Regarding the LES simulations, the addition of coherent turbulent structures via SEM treatment effectively eliminated the transition delay observed in previous work with hybrid RANS/LES methods. Excellent agreement is obtained for mean and fluctuating temperature contours in both magnitude and distribution throughout the flowfield. Very good agreement between the LES and experiment is also obtained for mean and fluctuating velocity components in regions of probe validity, particularly along the centerline of the jet. Off center of the jet, probe bias limits the confidence of the experimental measurements needed to validate the numerical solutions. Also, the effect of inflow turbulence from the tube is found to have a crucial effect on the resulting turbulent flow field. In contrast, the effect of the inflow turbulence from the duct is found to have a much more subtle effect.

The experimental challenges associated with making unsteady temperature measurements using $\mathrm{x}$ - $\mathrm{T}$ probes of different wire diameters is identified and sufficiently resolved to enable excellent agreement between experiment and numerics. The effect of low thin-wire cutoff frequency, an its associated loss of 
statistical variance, is shown to be adequately addressed using a compensation technique. The $1.2 \mu m$ diameter temperature sensor with compensation is shown to provide the best performance. The $\mathrm{x}$-T probes are also shown to have a limited domain of validity. The three-dimensional nature of the jet in a crossflow combined with the two-wire (x-wires) anemometry assumption of planar flow, leads to significant sensor error in regions that pose large flow angles relative to the assumed 2-D plane. Consequently, the U-W probe is found to inaccurate in most of the jet and wake region due to significant vertical velocity components. The $\mathrm{U}-\mathrm{V}$ probe is found to be inaccurate only off the center plane where large spanwise velocity gradients exist. The velocity fluctuations do not appear to be as adversely affected by the mean flow bias, as the mean flow, and its biasing effects, are largely removed in the statistical averaging process. The large probe volume is also a primary limitation of the xT-wire probe accuracy. The large probe volume is a result of the length of the sensing wires, and the separation between wires. This limits the size of the flow features which can be resolved.

Heat flux measurements are adversely affected by both the frequency limits of temperature measurements as well as the large probe volumes. The heat flux is measured though the correlation of velocity and temperature signals. The miss-match in temperature and velocity sampling frequency will contaminate the time correlation needed to measure the heat flux. A similar effect is seen when numerical compensation methods are applied to the CCA temperature signal, noise is added to the signal to adjust for the low pass filtering without knowledge of the signal correlation. The measured heat flux made is a two point correlation due to the moderate wire separation within the probe volume. At various locations within the flow different sensing wires may be in fundamentally different flow regions due to the wire separation.

The validation of LES simulations of a heated jet in a crossflow have been validated against experiment, for mean and fluctuating velocities and temperature. The difficulties which exist for the direct measurement of turbulent heat fluxes have limited the ability to directly validate the LES heat flux predictions against the experiment. However, the excellent agreement of velocity and temperature profiles with the experimental measurements individually give large confidence in the validity of the LES heat flux predictions. Further work will be done to apply a representative two point heat flux correlation to the LES results. This should confirm the current deficiencies which exist in the measurements of turbulent heat fluxes. Work should also continue to be done to improve heat flux measurements. 


\section{References}

${ }^{1}$ Goldstein, R. J., Eckert, E. R. G., and Ramsey, J. W., "Film Cooling with Injection Through Holes: Adiabatic Wall Temperatures Downstream of a Circlular Hole," Engineering for Power, Vol. 90, 1968.

${ }^{2}$ Bogard, D. G. and Thole, K. A., "Gas Turbine Film Cooling," Journal of Propulsion and Power, Vol. 22, No. 2, 2006.

${ }^{3}$ Mahesh, K., "The Interaction of Jets with Crossflow," Annual Review of Fluid Mechanics, Vol. 45, No. 1, 2013, pp. 379407.

${ }^{4}$ Ruggles-Wrenn, M. B. and Jones, T. P., "Tension-Compression Fatigue of a SiC/SiC Ceramic Matrix Composite at Elevated Temperature," Journal of Engineering for Gas Turbines and Power, Vol. 134, 2012.

${ }^{5}$ Deng, B., Wu, W., and Xi, S., "A near-wall two-equation heat transfer model for wall turbulent flows," International Journal of Heat and Mass Transfer, Vol. 44, No. 4, 2001, pp. 691-698.

${ }^{6}$ Karcz, M. L. and Badur, J., "A turbulent heat flux two-equation closure based on the $\{\mathrm{V} 2 \mathrm{~F}\}$ turbulence model," Task quarterly, Vol. 7, No. 5, 2003, pp. 375-387.

${ }^{7}$ Andreopoulos, J. and Rodi, W., "Experimental Investigation of Jets in a Crossflow," Journal of Fluid Mechanics, Vol. 138, 1984, pp. 93-127.

${ }^{8}$ Fric, T. F. and Roshko, A., "Vortical Structure in the Wake of a Transverse Jet," Journal of Fluid Mechanics, Vol. 279, 1994, pp. 1-47.

${ }^{9}$ Coussement, A., Gicquel, O., and Degrez, G., "Large Eddy Simulation of a Pulsed Jet in Cross-Flow," Journal of Fluid Mechanics, Vol. 695, 2012, pp. 1-34.

${ }^{10}$ Cortelezzi, L. and Karagozian, A. R., "On the Formation of the Counter-Rotating Vortex Pair in Transverse Jets," Journal of Fluid Mechanics, Vol. 446, 2001, pp. 347-373.

${ }^{11}$ Wassel, A. and Catton, I., "Calculation of Turbulent Boundary Layers Over Flat Plates with Different Phenomenological Theories of Turbulence and Variable Turbulent Prandtl Number," Int. J. Heat Mass Transfer, Vol. 16, 1973, pp. $1547-1563$.

${ }^{12}$ Bradshaw, P., Launder, B., and Lumley, J., "Collaborative Testing of Turbulence Models," AIAA Paper 1991-0215, 1991.

${ }^{13}$ Sommer, T., So, R., and Zhang, H., "Near-Wall Variable-Prandlt-Number Turbulence Model for Compressible Flow," AIAA Journal, Vol. 31, 1993, pp. 27-35.

${ }^{14}$ Ivanova, E., Noll, B., Domenico, M. D., and Aigner, M., "Improvement and Assesment of RANS Scalar Transport Models for Jets in Crossflow," AIAA Paper 2008-565, 2008.

${ }^{15}$ Borghi, M., Thurman, D., Poinsatte, P., Werenet, M., and Engblom, W., "Preliminatry Experimental and Numerical Investigation of," AIAA Paper 2017-0414, 2017.

${ }^{16}$ Johnson, P. and Kapat, J., "Large-Eddy Simulations of a Cylindrical Film Cooling Hole," Journal of Thermophysics and Heat Transfer, Vol. 27, 2013, pp. 255-273.

${ }^{17}$ Renze, P., Schroder, W., and Meinke, M., "Large-Eddy Simulation of Film Cooling Flows at Density Gradients," International Journal of Heat and Fluid Flow, Vol. 29, 2008, pp. 18-34.

${ }^{18}$ Lund, T. S., Wu, X., and Squires, K. D., "Generation of Turbulent Inflow Data for Spatially-Developing Boundary Layer Simulations," Journal of Computational Physics, Vol. 140, No. 2, 1998, pp. 233-258.

${ }^{19}$ Jarrin, N., Addad, Y., and Laurence, D., "Synthetic Turbulent Inflow Conditions for Large Eddy Simulation," Proceedings, 4th International Turbulence, Heat and Mass Transfer Conference, Antalya Turkey, 2003.

${ }^{20}$ Klein, M., "A Digital Filter based Generation of Inflow Data for Spatially Devleoping Direct Numerical or Large Eddy Simulations," Journal of Computational Physics, Vol. 186, 12003, pp. 233-258.

${ }^{21}$ Jarin, N., Benhamadouche, S., Laurence, D., and Prosser, R., "A synthetic-eddy-method for generating inflow conditions for large-eddy simulations," The International Journal of Heat and Fluid Flow, Vol. 27, 2016.

${ }^{22}$ Jarrin, N., Prosser, R., Uribe, J. C., Benhamadouche, S., and Laurence, D., "Reconstruction of turbulent fluctuations for hybrid RANS/LES simulations using a Synthetic-Eddy Method," International Journal of Heat and Fluid Flow, Vol. 30, No. 3, 2009, pp. 435-442.

${ }^{23}$ Poletto, R., Craft, T., and Revell, A., "A New Divergence Free Synthetic Eddy Method for the Reproduction of Inlet Flow Conditions for LES," Flow Turbulence and Combustion, Vol. 91, 2013.

${ }^{24}$ Patruno, L. and Ricci, M., "On the generation of synthetic divergence-free homogeneous anisotropic turbulence," Computer Methods in Applied Mechanics and Engineering, Vol. 315, 2017.

${ }^{25}$ Skillen, A., Revell, A., and Craft, T., "Accuracy and efficiency improvements in synthetic eddy methods." International Journal of Heat and Fluid Flow, Vol. 62, 2016, pp. 386-394.

${ }^{26}$ Muldoon, F. and Acharya, S., "Analysis of $k-\epsilon$ Budgets for Film Cooling Using Direct Numerical Simulation," AIAA Journal, Vol. 44, 2000.

${ }^{27}$ Burdet, A., Abhari, R., and Rose, M., "Modeling of Film Cooling-Part II: Model for use in Three-Dimensional Computational Fluid Dynamics," Journal of Turbomachinery, Vol. 129, 2007.

${ }^{28}$ Crabb, D., Durao, D. F. D., and Whitelaw, J. H., "A Round Jet Normal to a Crossflow," Journal of Fluid Engineering, Vol. 103, 1981, pp. 142-153.

${ }^{29}$ Tagawa, M., Kato, K., and Ohta, Y., "Response compensation of fine-wire temperature sensors," Review of Scientific Instruments, Vol. 76, No. 9, 2005.

${ }^{30}$ Smits, A. J., Perry, A. E., and Hoffmann, P. H., "The response to temperature fluctuations of a constant-current hot-wire anemoneter," Journal of Physics E: Scientific Instruments, Vol. 11, 1978.

${ }^{31}$ Khalkhal, H. K., Measurement of Temperature Dissipation in a Turbulent Wake Using Cold Wires with Numerical Compensation, Ph.D. thesis, University of Laval, Quebec, Canada, 1997.

${ }^{32}$ Arwatz, G., Bahri, C., Smits, A. J., and Hultmark, M., "Dynamic Calibration and modeling of a cold wire for temperature measurement," Measurement Science and Technologly, Vol. 24, 2013. 
${ }^{33}$ Thurman, D., Poinsatte, P., Ameri, A., Culley, D., Raghu, S., and Shyam, V., "Investigation of Sprial and Sweeping Holes," Journal of Turbomachinery, Vol. 138, 2016.

${ }^{34}$ Schlatter, P., Orlu, R., Li, Q., Brethouwer, G., Fransson, J., Johansson, A., Alfredsson, P., and Henningson, D., "Turbulent Boundary Layers up to $R e_{\Theta}=2500$ studied through simulation and experiment," Physics of Fluids, Vol. $21,2009$. 


\section{Figures}

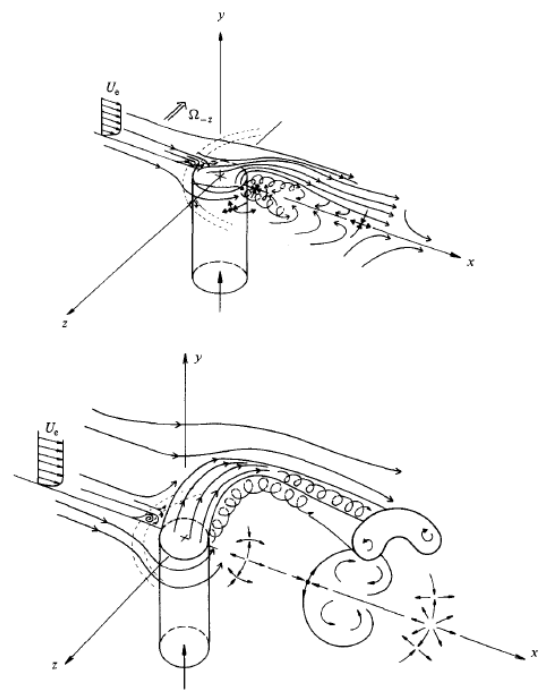

(a) Effect of blowing ratio on a jet in crossflow. (top) Lower $B R$. (bottom) Higher BR. From Andreopoulos (1984).

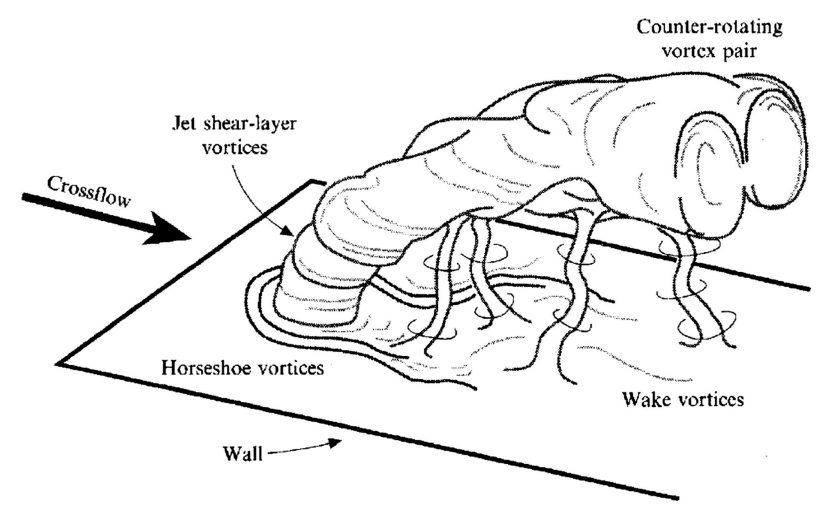

(b) Near field region of a jet in crossflow. From Fric (1994).

Figure 1. Schematic depictions of jets in crossflows.

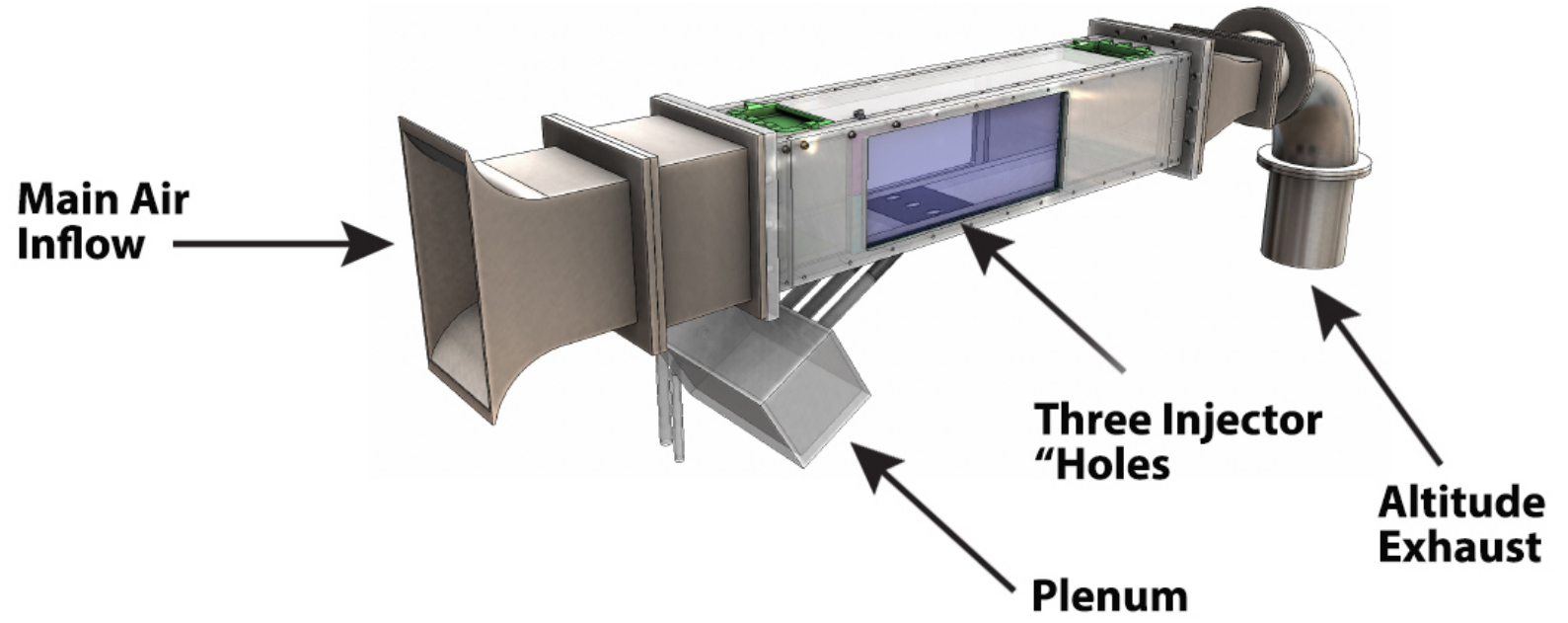

Figure 2. SW-6 Facility in Center Wide Exhaust Configuration. From Wernet et. al. 


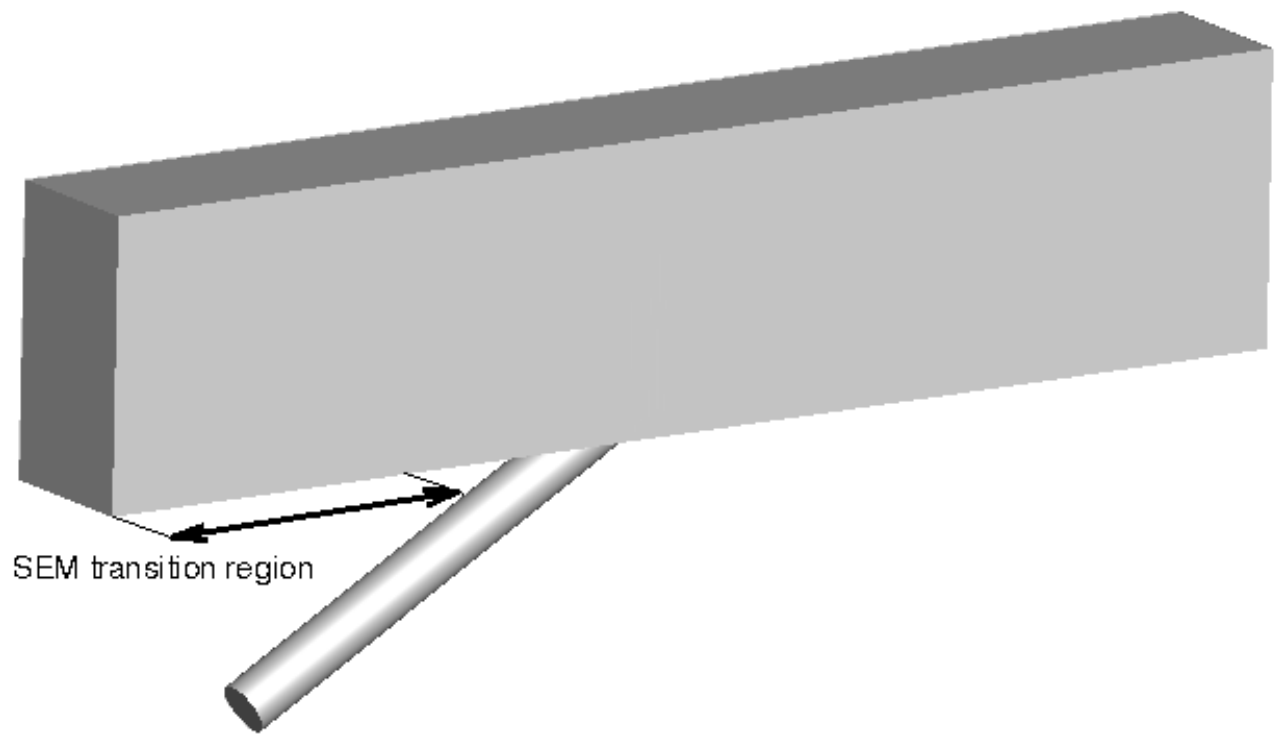

Figure 3. Computational domain, highlighting the extra inflow domain used for SEM transition.

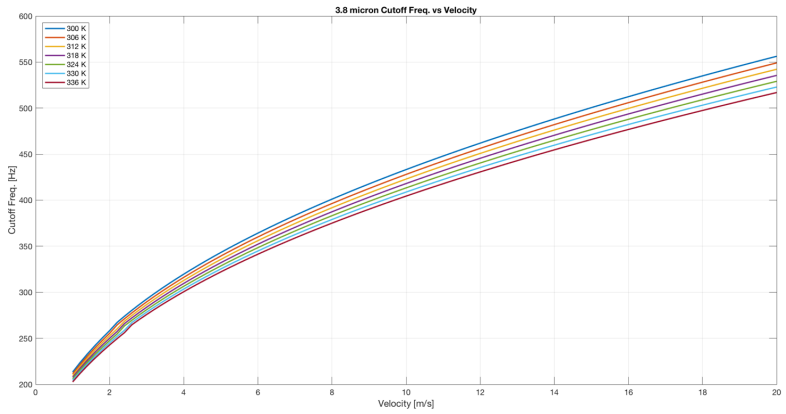

(a) 3.8 mum wire

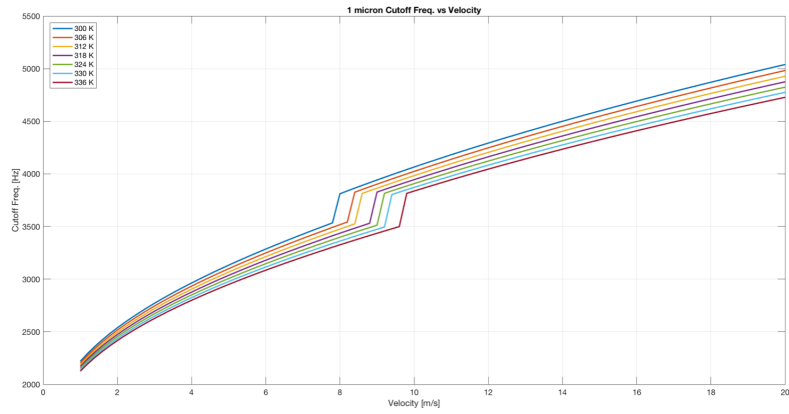

(b) $1.2 \mathrm{mum}$ wire

Figure 4. Family of cutoff frequency curves for fine wire thermometers used in the current work
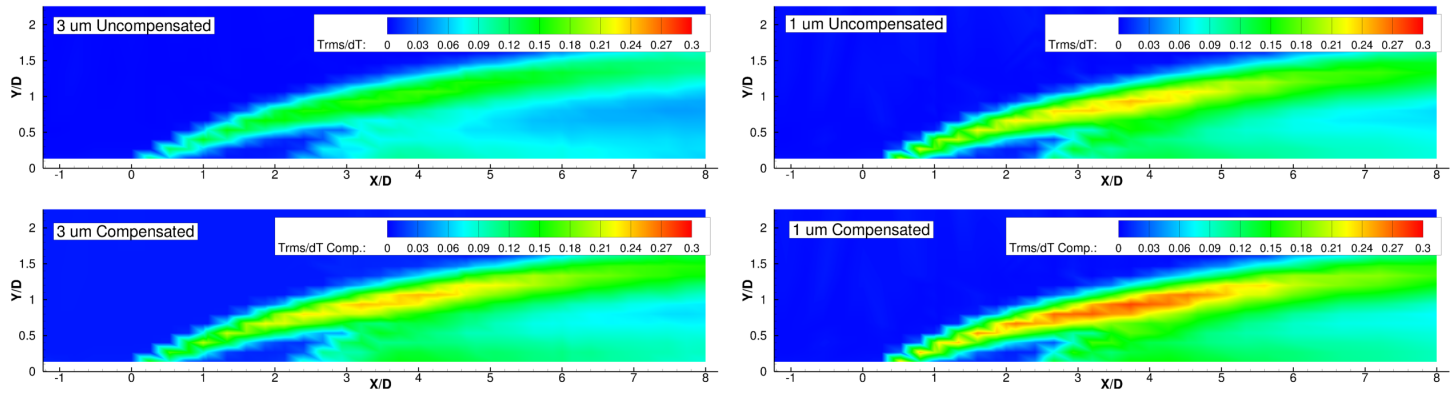

Figure 5. Effects of CCA compensation on $3.8 \mu m$ and $1.2 \mu m$ wires 


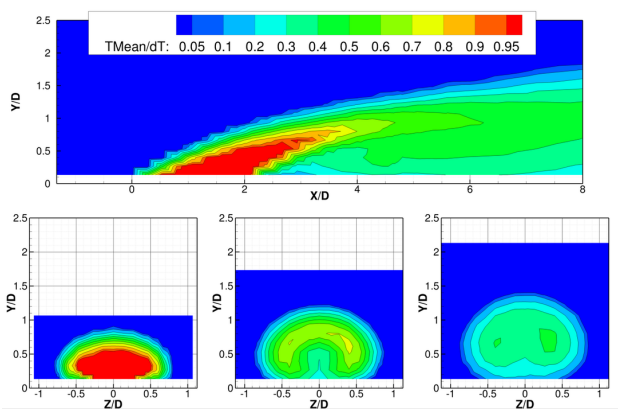

(a) UV-Probe Normalized Mean Temperature

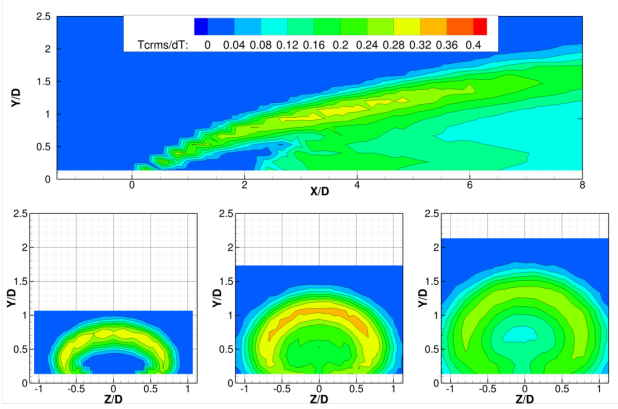

(c) UV-Probe Normalized Temperature variance

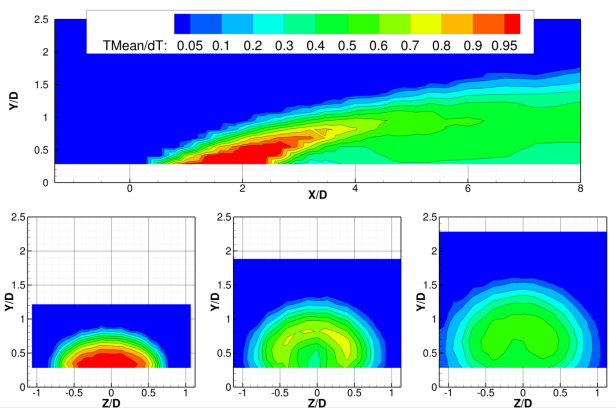

(b) UW-Probe Normalized Mean Temperature
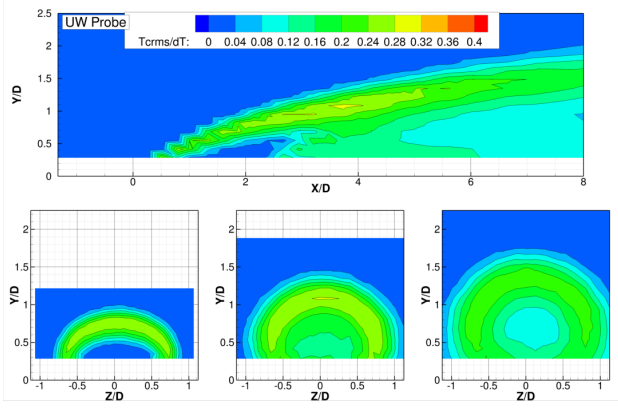

(d) UW-Probe Normalized Temperature variance

Figure 6. Normalized temperature measurements, Mean and variance, with UV- and UW- xT probes at Z/D $=0.00, X / D=2.00, X / D=4.00, X / D=6.00$

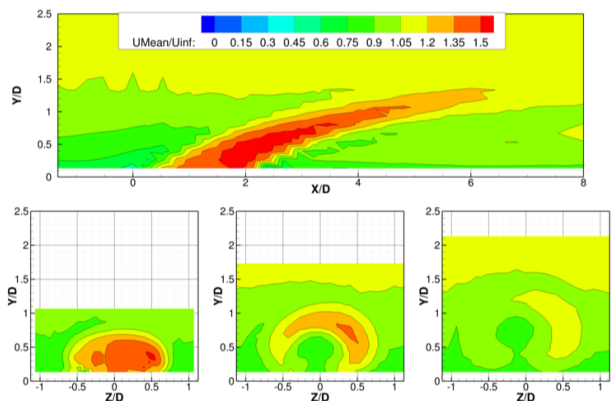

(a) UV-Probe mean streamwise velocity
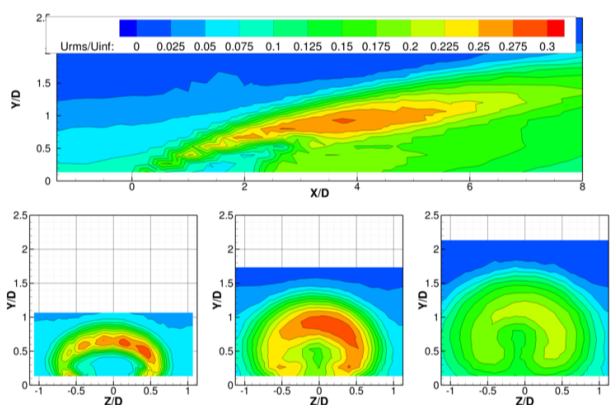

(c) UV-probe streamwise velocity fluctuations
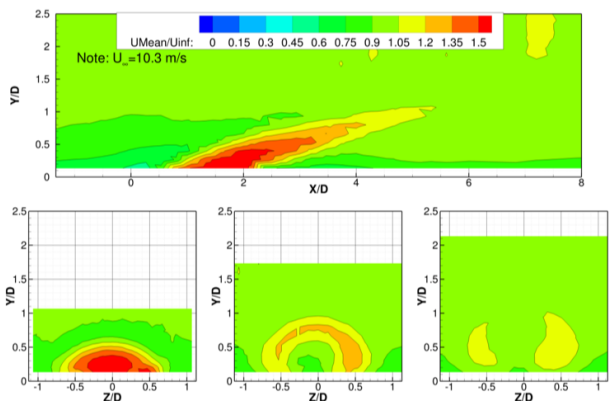

(b) UW-Probe mean streamwise velocity
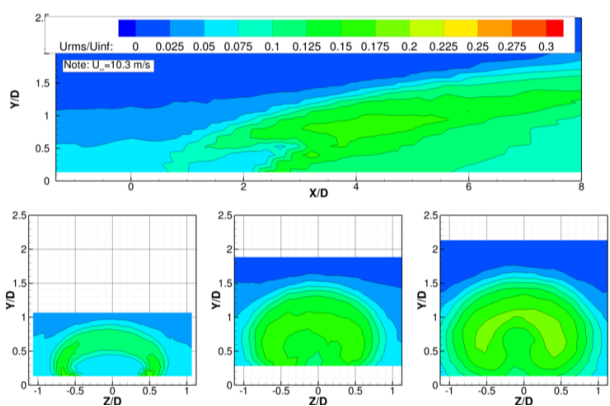

(d) UW-probe streamwise velocity fluctuations

Figure 7. Normalized temperature measurements, Mean and variance, with UV- and UW- xT probes at Z/D $=0.00, \mathrm{X} / \mathrm{D}=2.00, \mathrm{X} / \mathrm{D}=4.00, \mathrm{X} / \mathrm{D}=6.00$ 

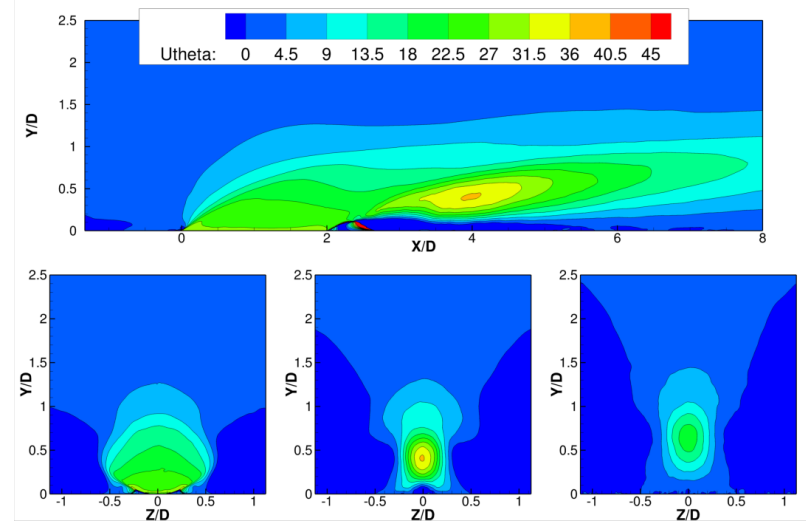

(a) No SEM in the tube
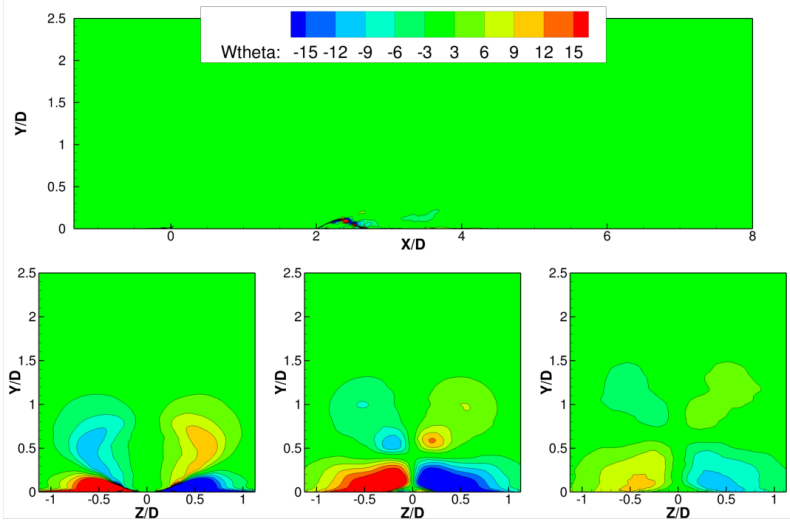

(b) SEM in the tube

Figure 8. Local flow angles $\Theta_{u}$ and $\Theta_{w}$ computed from the Full-SEM LES

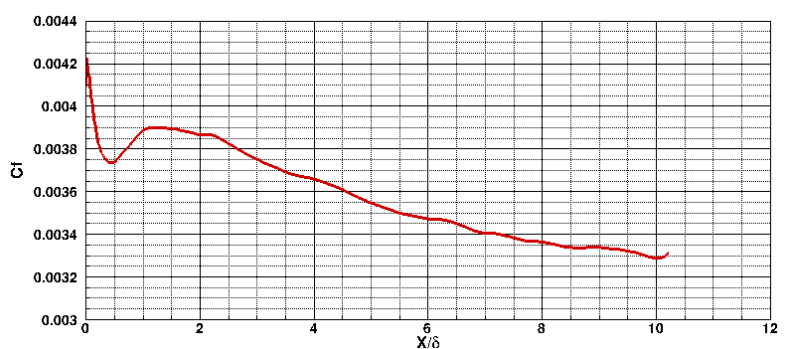

(a) Span averaged friction coefficient as a function of axial distance downstream.

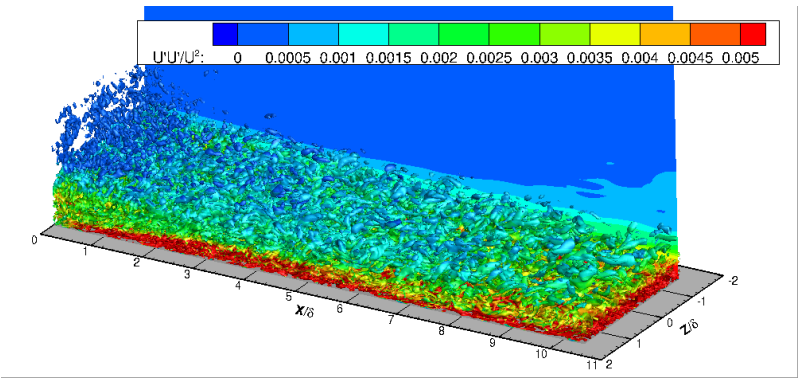

(b) Iso-countour of $\mathrm{Q}$-Criterion $(\mathrm{Q}=50,000)$ colored by the noralized axial Reynolds stress.

Figure 9. Flat plate with SEM inflow region 


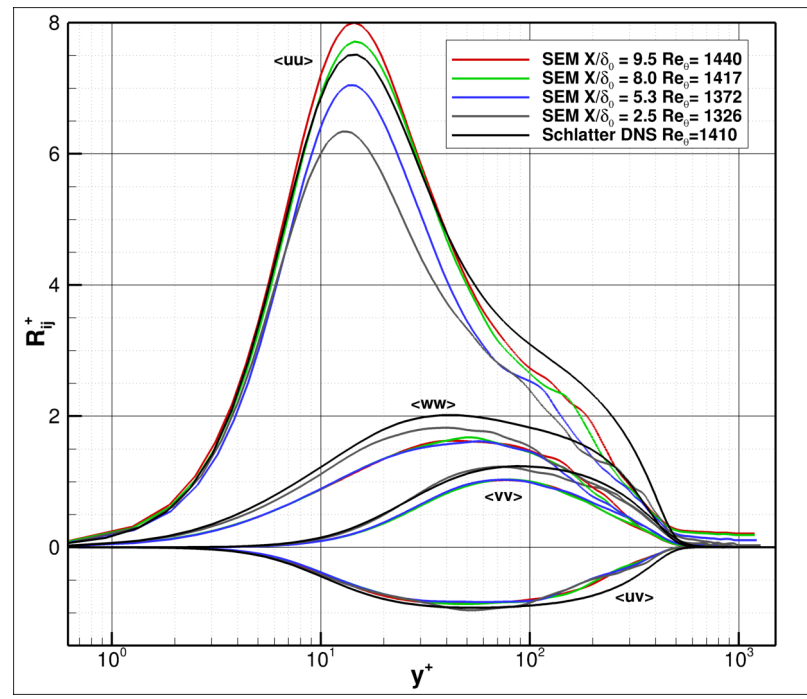

Figure 10. Variation of $R_{i j}$ at different locations downstream of the SEM inlet. Momentum thickness Reynolds number provided for reference, along with DNS data from Schlatter. ${ }^{34}$
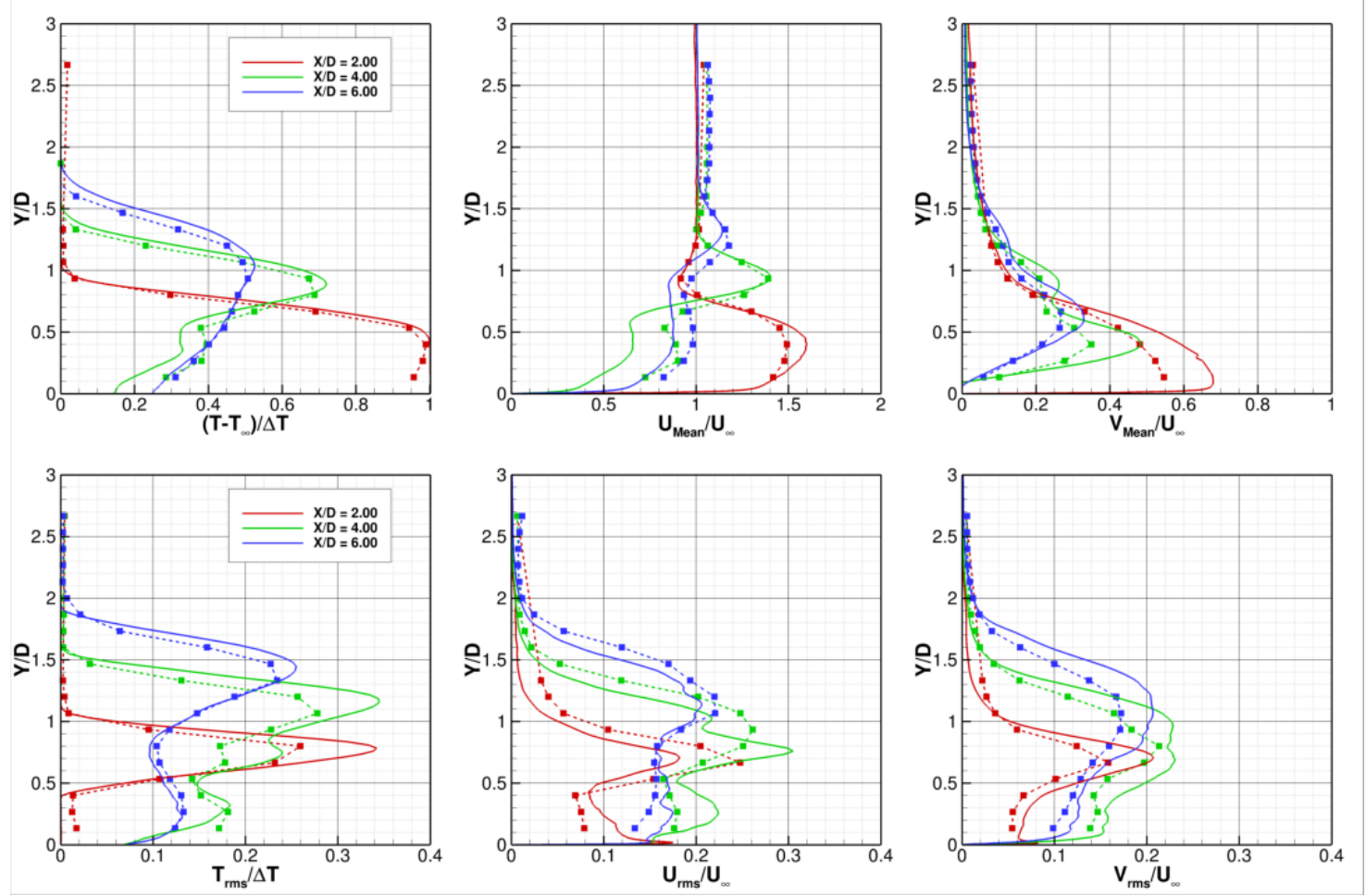

Figure 11. Full-SEM LES (solid line) compared with CCA and CTA data taken with the UV-probe (symbols) 

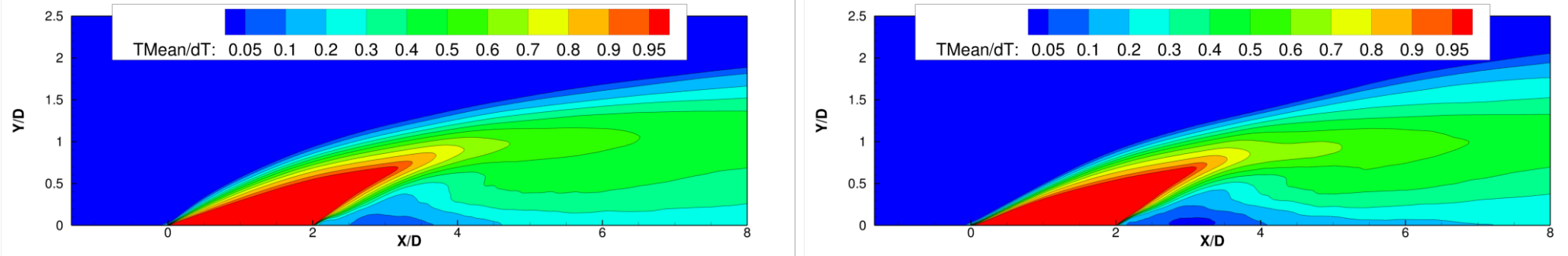
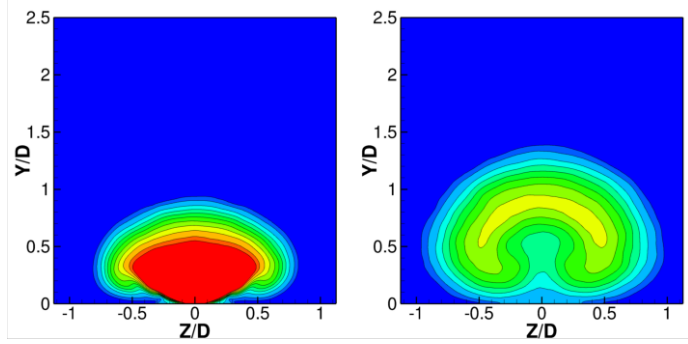

(a) Full-SEM
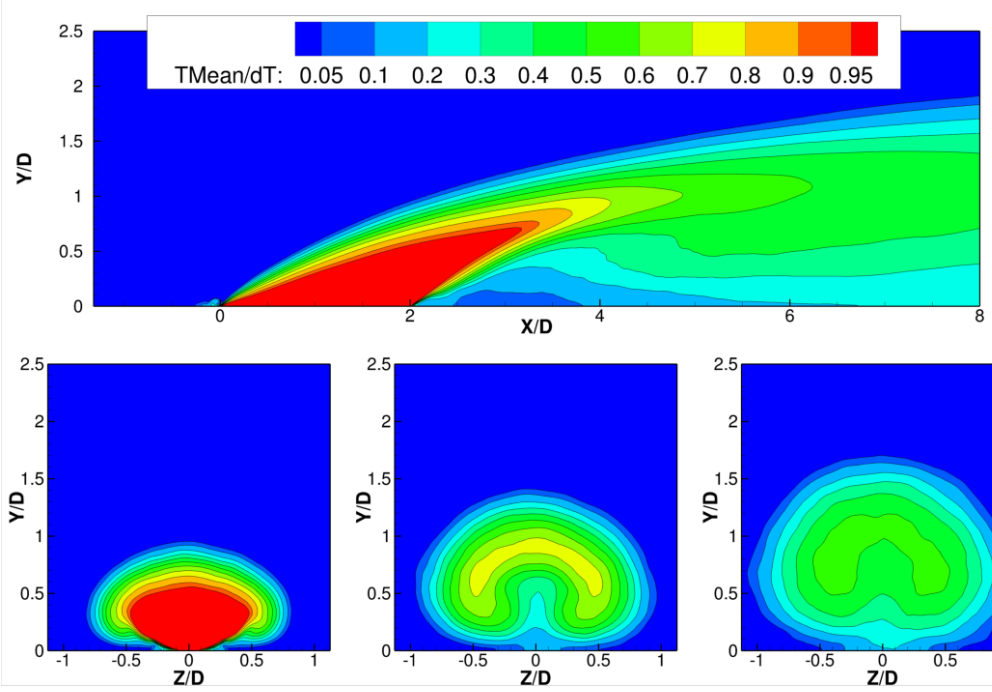

(c) Tube-SEM
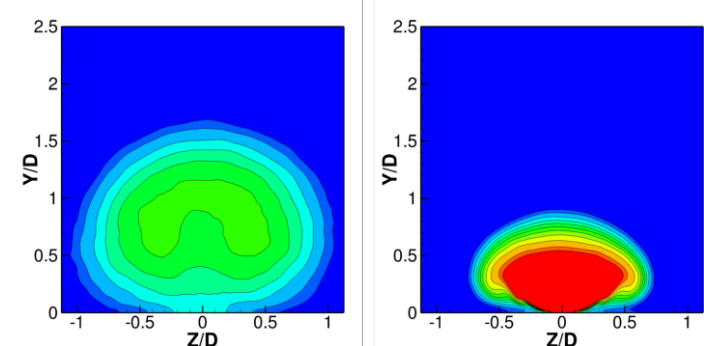

zio

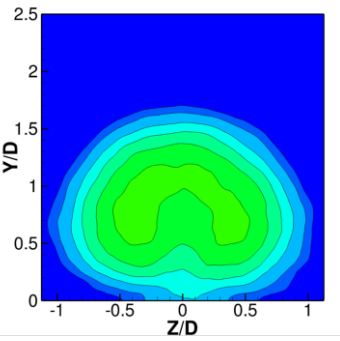

Figure 12. Normalized Mean temperature profiles from LES and U-V xT-wire probe

${ }^{-0.5}$ z/D $^{0.5}$

(b) Tunnel-SEM
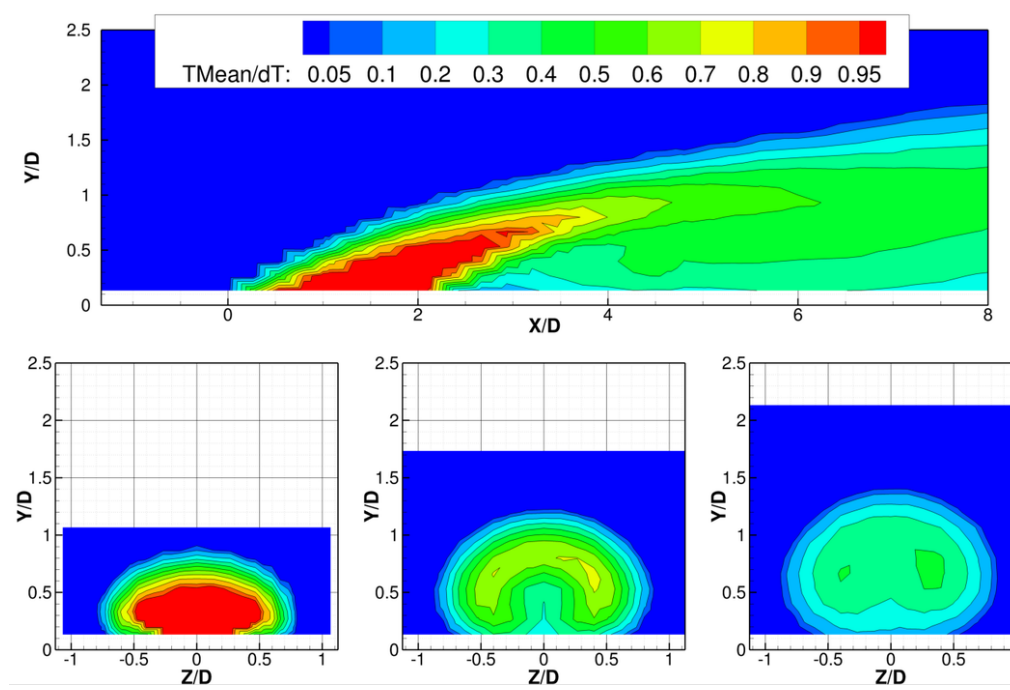

(d) U-V Probe

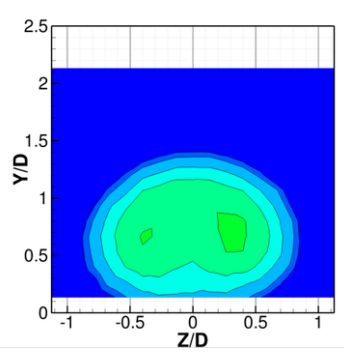

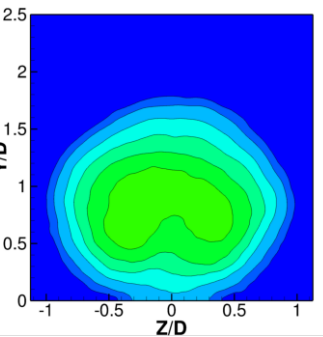

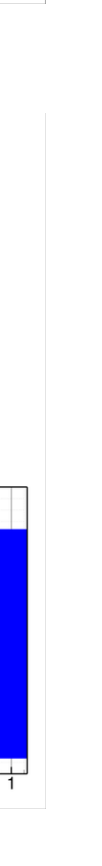



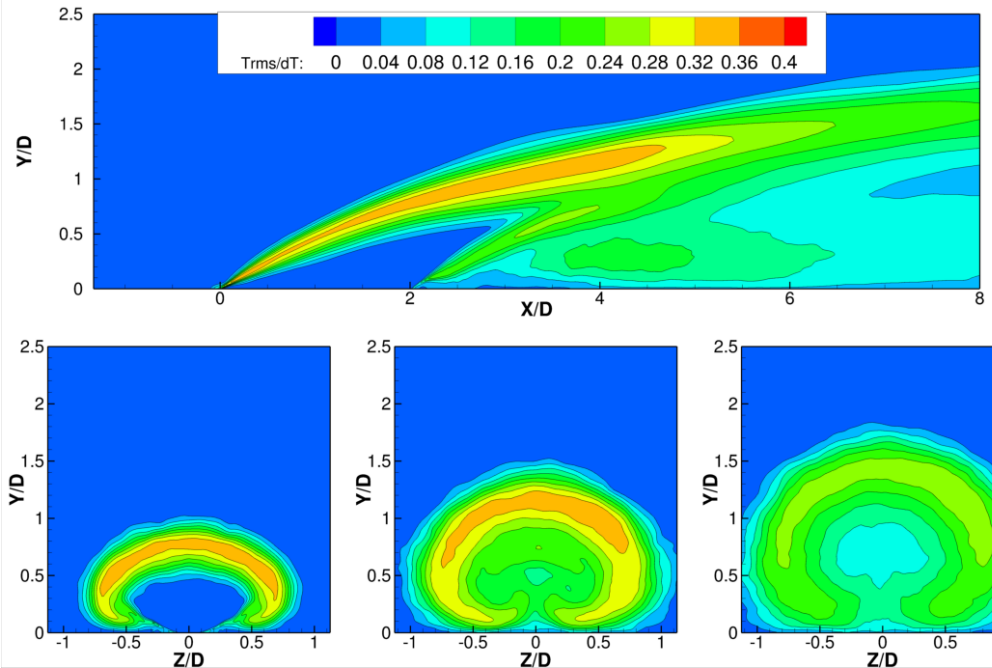

(a) Full-SEM

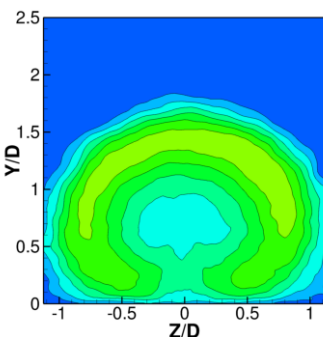

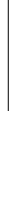
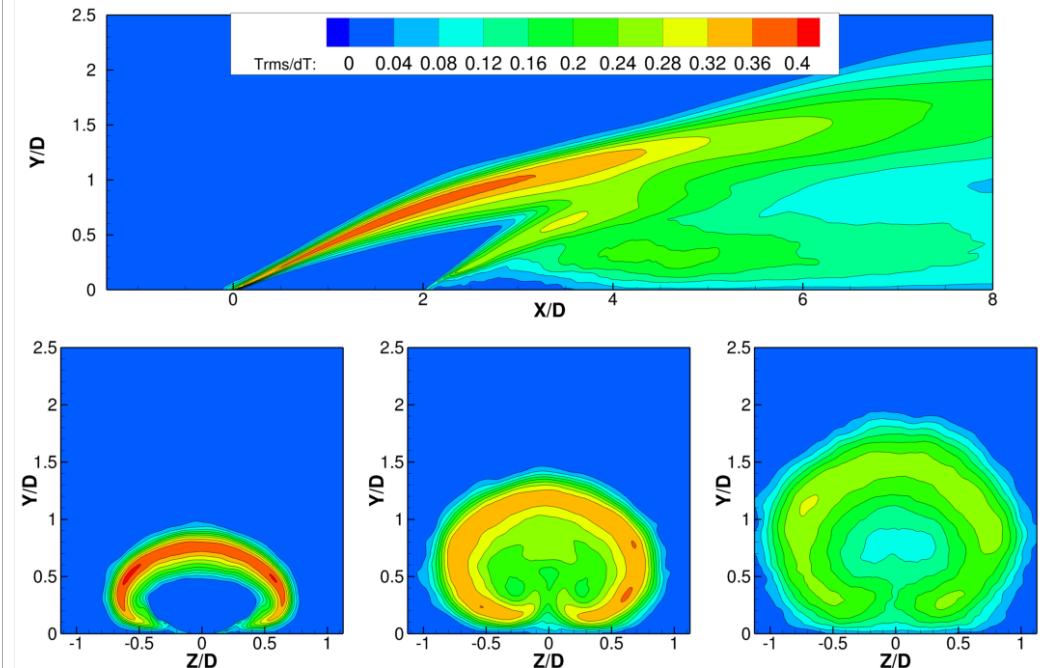

(b) Tunnel-SEM
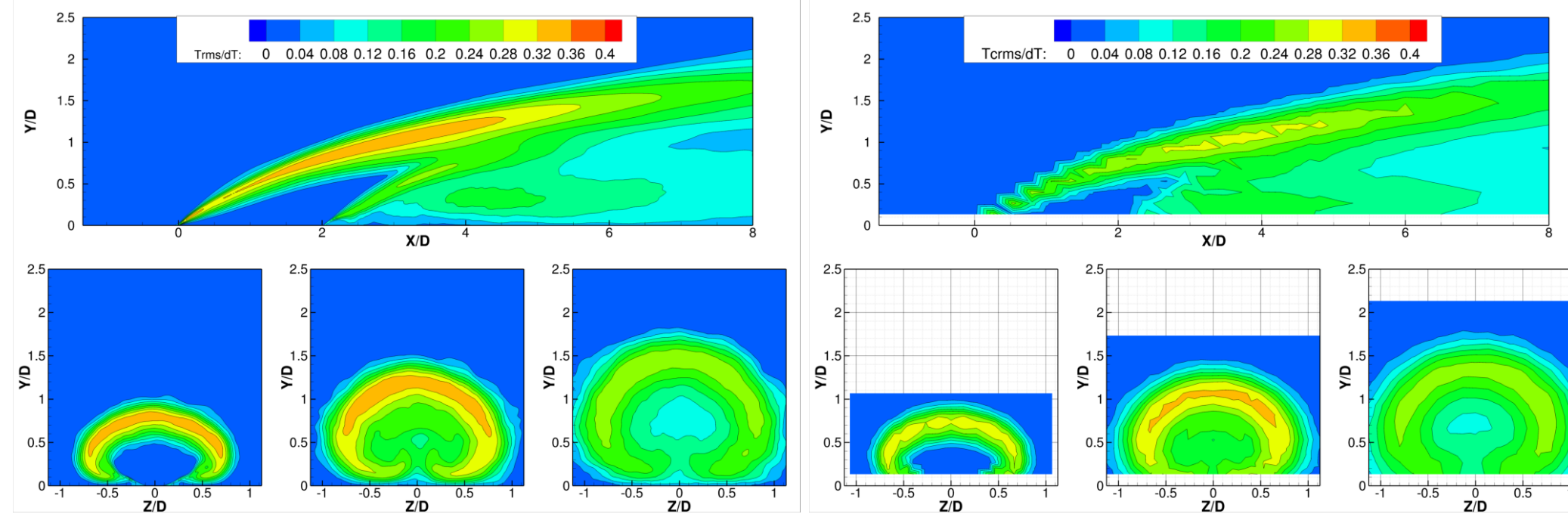

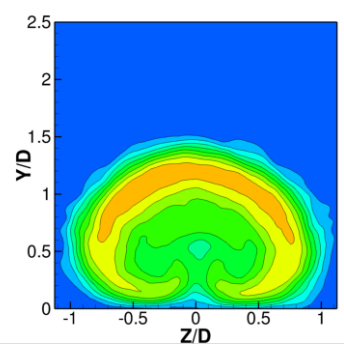

(c) Tube-SEM
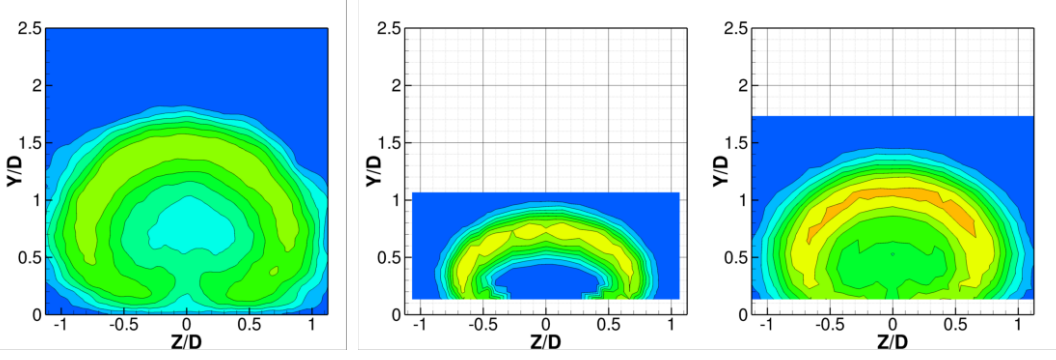

(d) U-V Probe

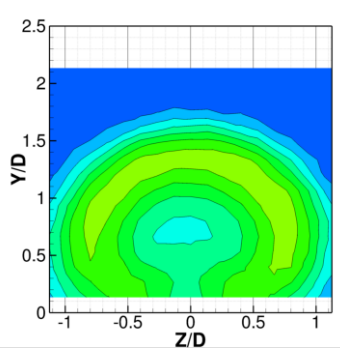

Figure 13. Room-mean-squared temperature fluctuations normalized by temperature difference. 

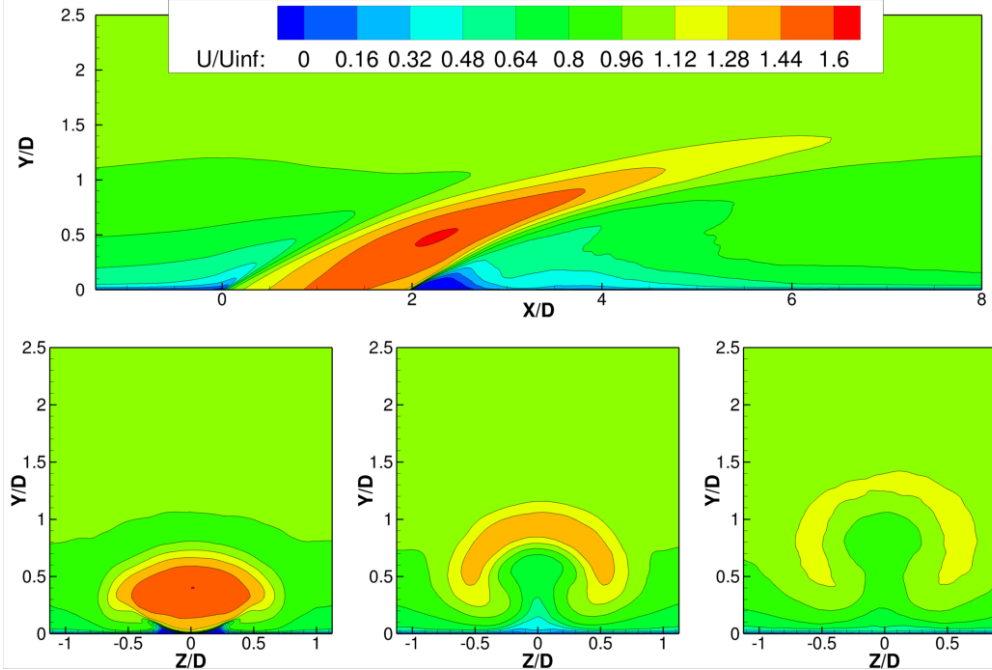

(a) Full-SEM

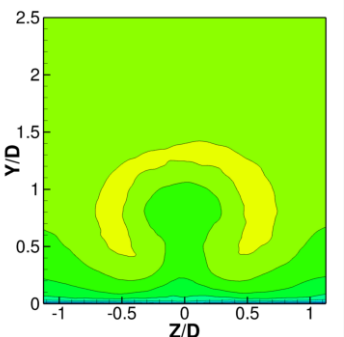

id

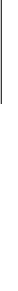
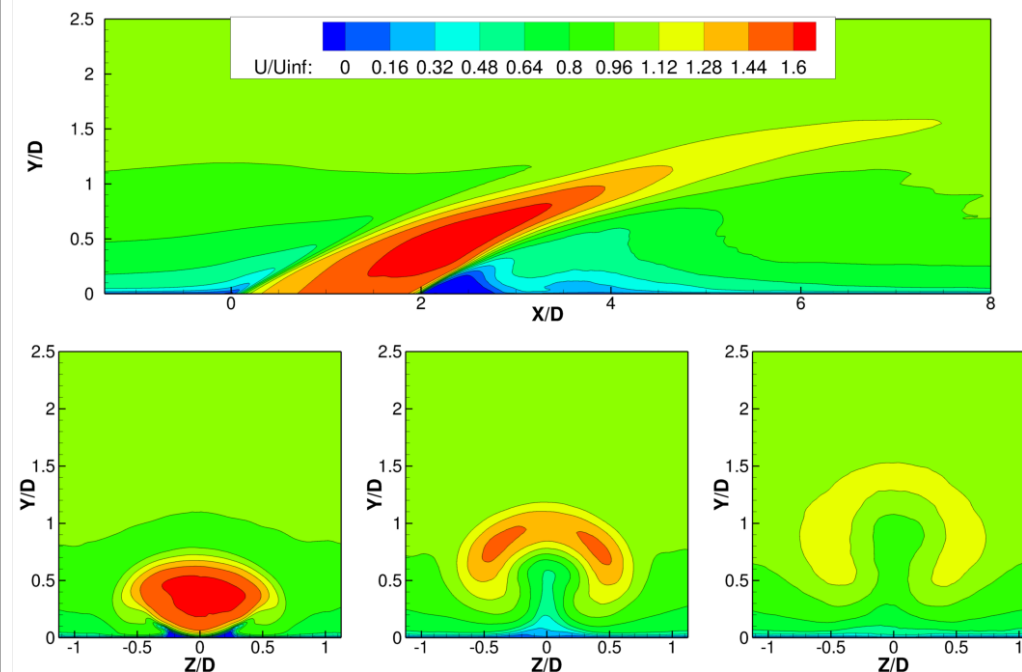

(b) Tunnel-SEM

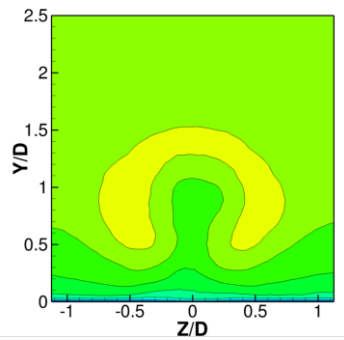

ZiD
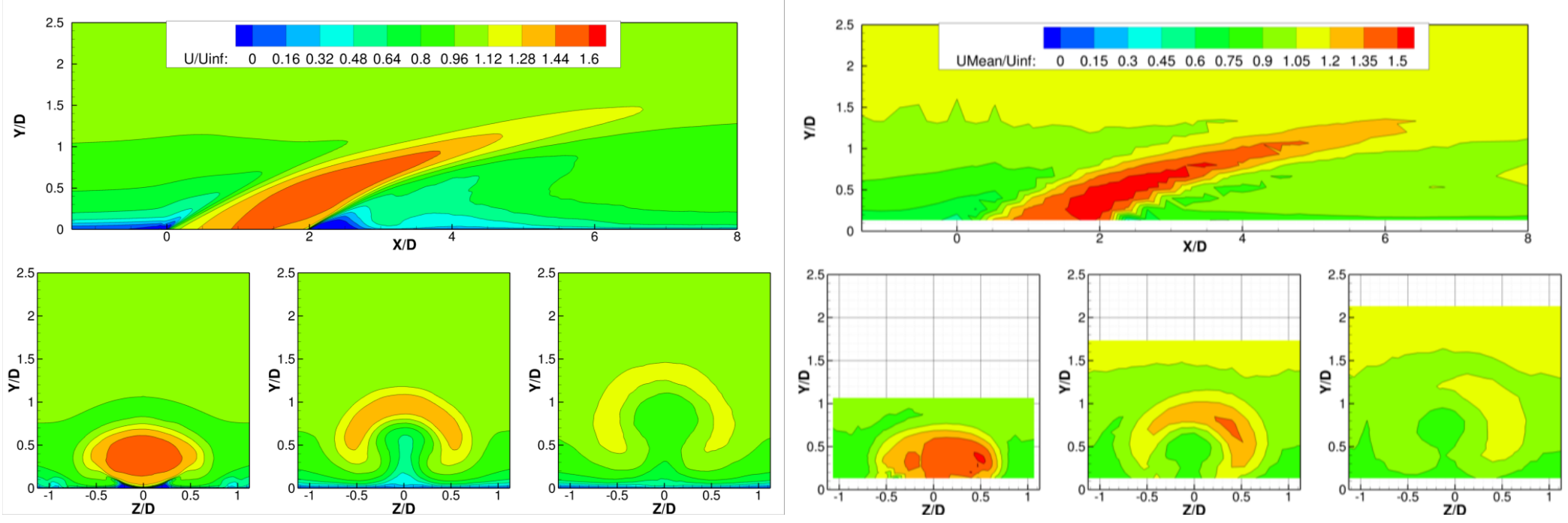

(c) Tube-SEM

(d) U-V Probe

Figure 14. Mean axial velocity contours from LES and U-V xT-wire Probe 

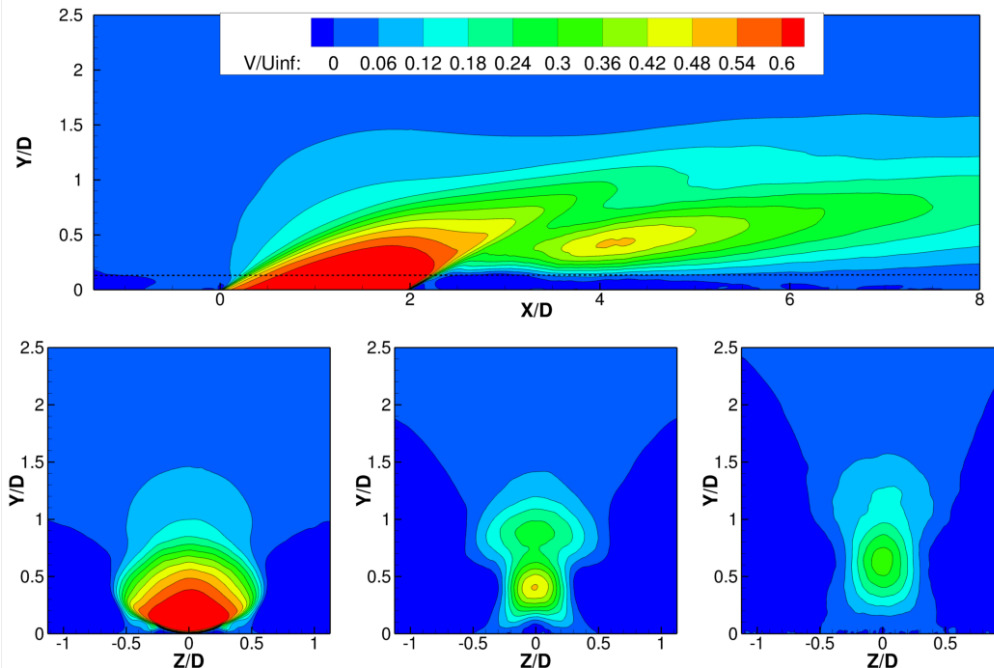

(a) Full-SEM
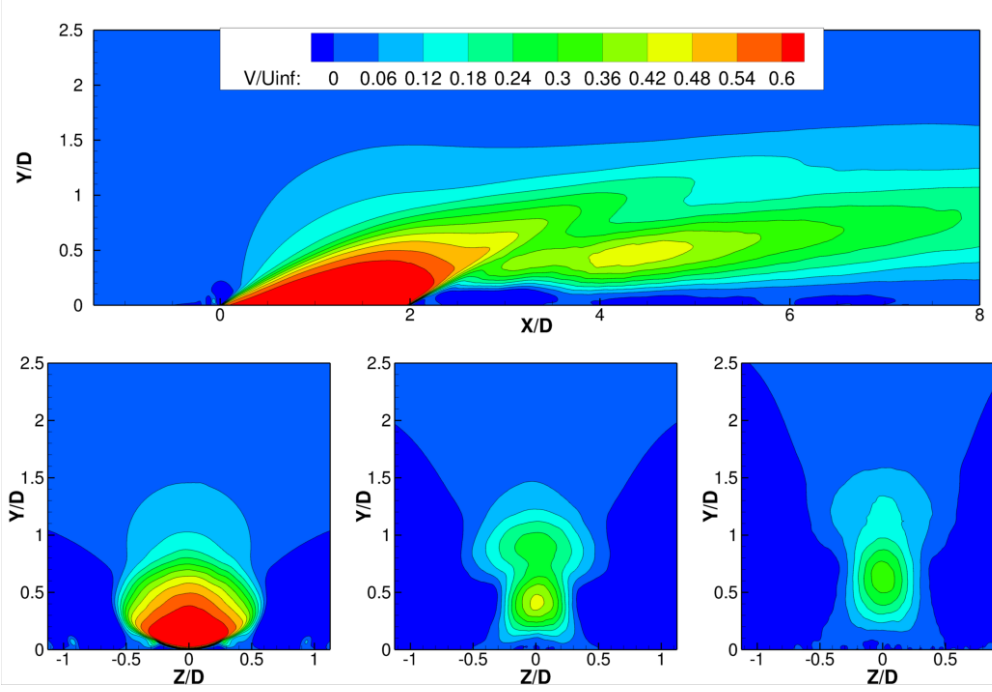

(c) Tube-SEM
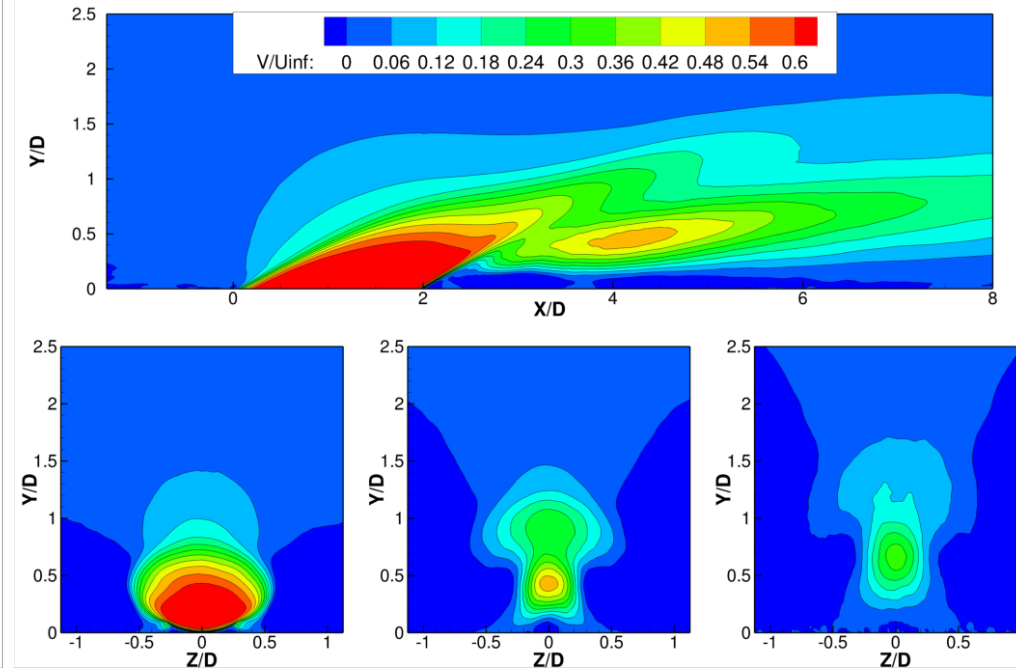

(b) Tunnel-SEM
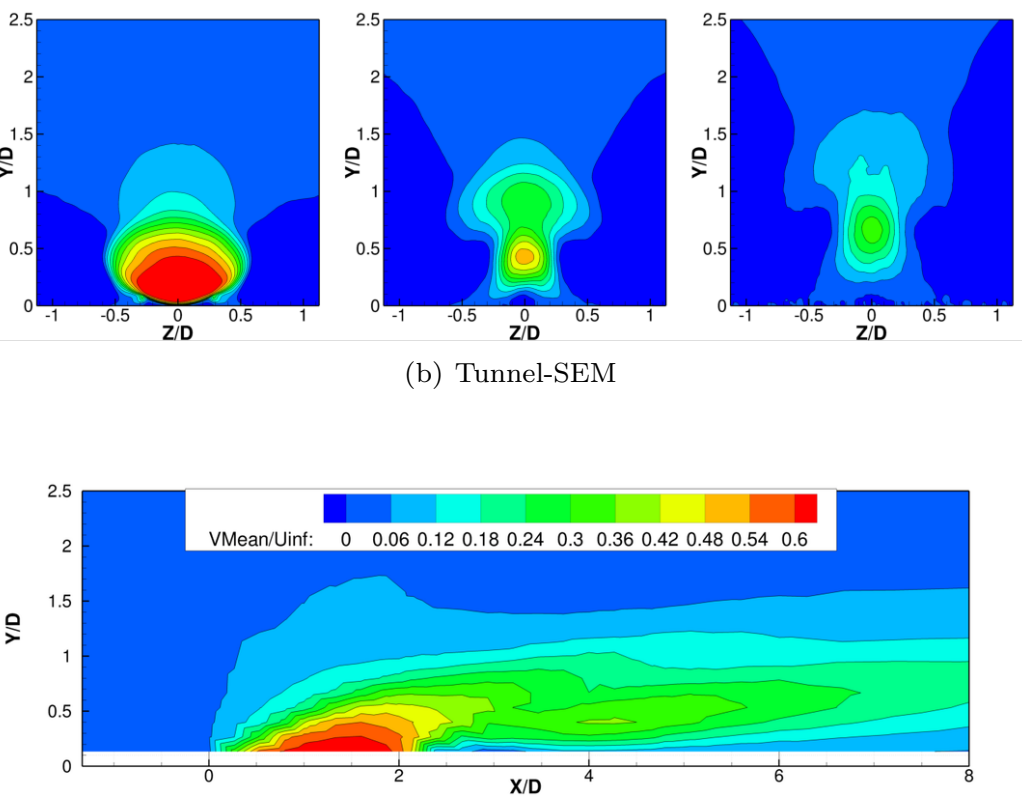
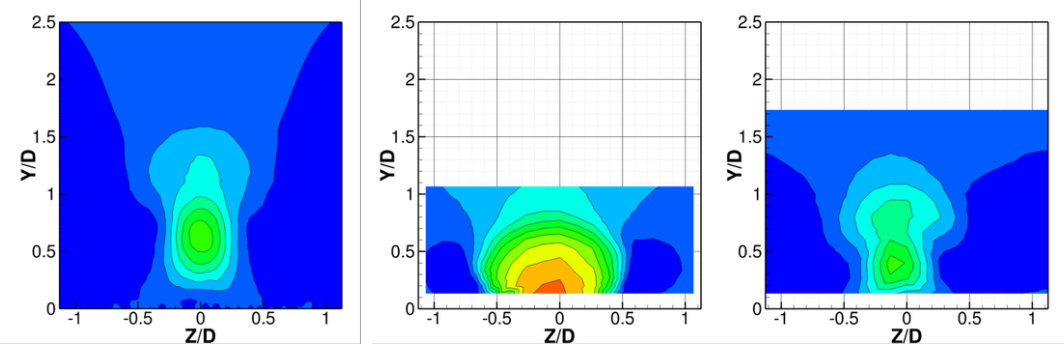

(d) U-V Probe

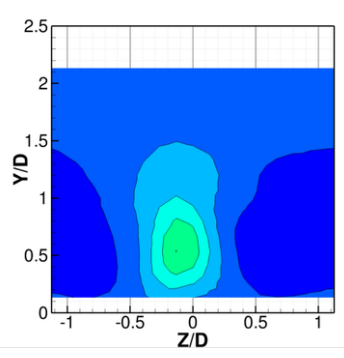

Figure 15. Mean vertical velocity contours from LES and U-V xT-wire Probe 


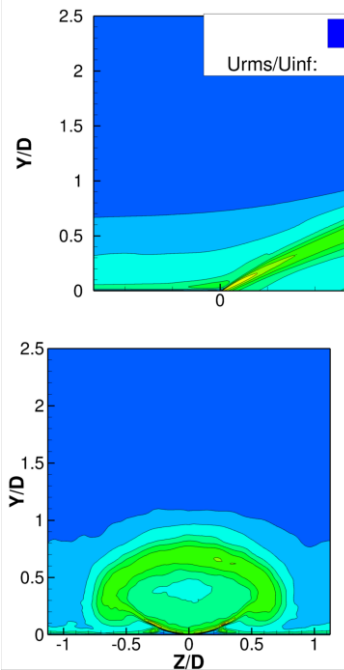

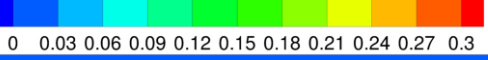

(a) Full-SEM

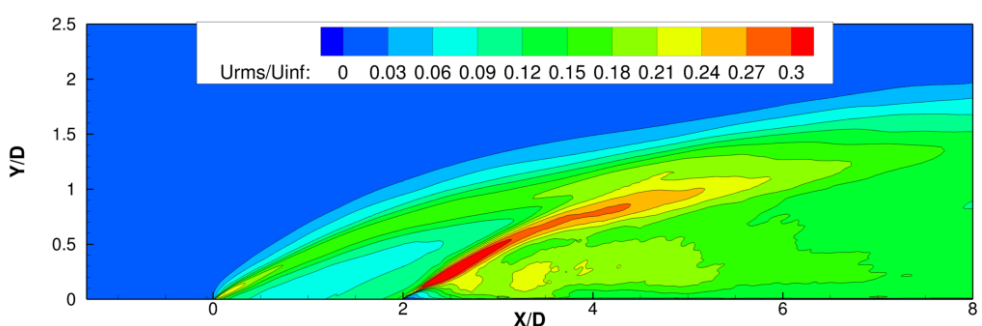

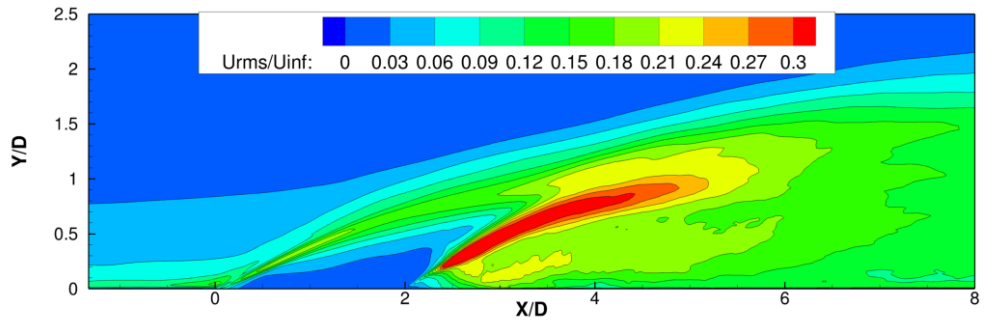
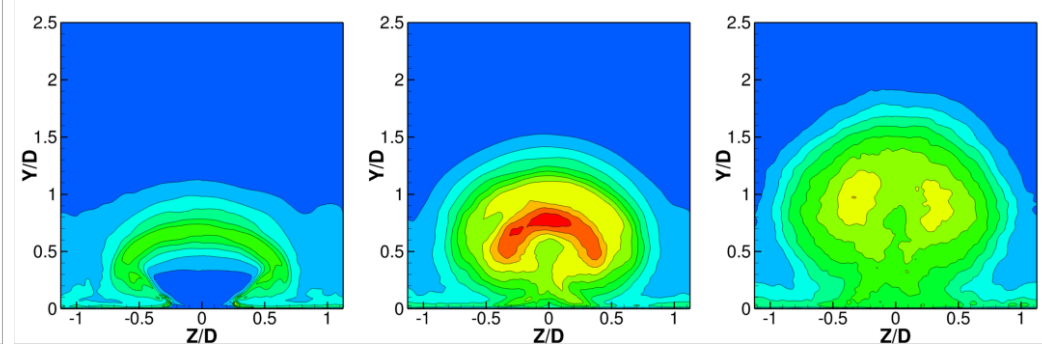

(b) Tunnel-SEM

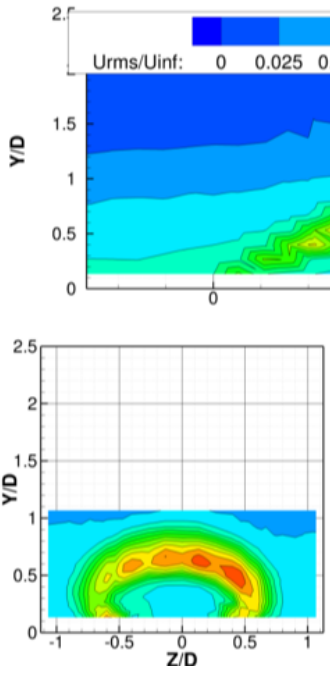

(c) Tube-SEM

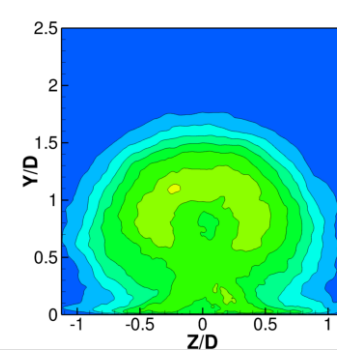

Z/D
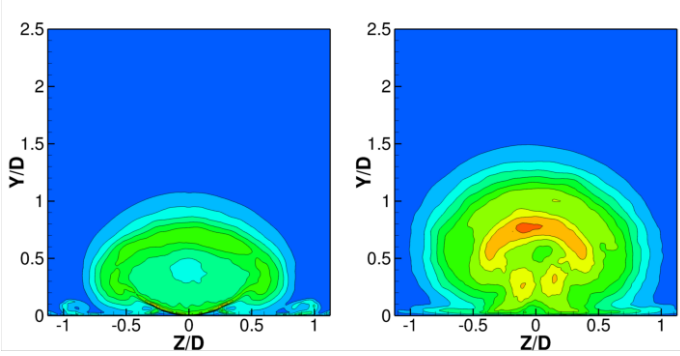

Figure 16. Root-mean-squared streamwise velocity fluctuations contours from LES and U-V xT-wire Probe 


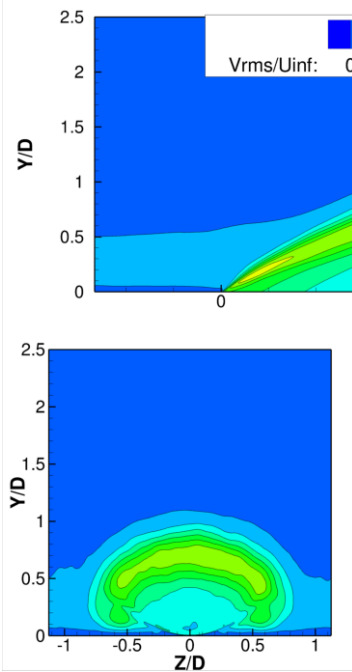

Z/D $\begin{array}{lllllllllll}0 & 0.03 & 0.06 & 0.09 & 0.12 & 0.15 & 0.18 & 0.21 & 0.24 & 0.27 & 0.3\end{array}$
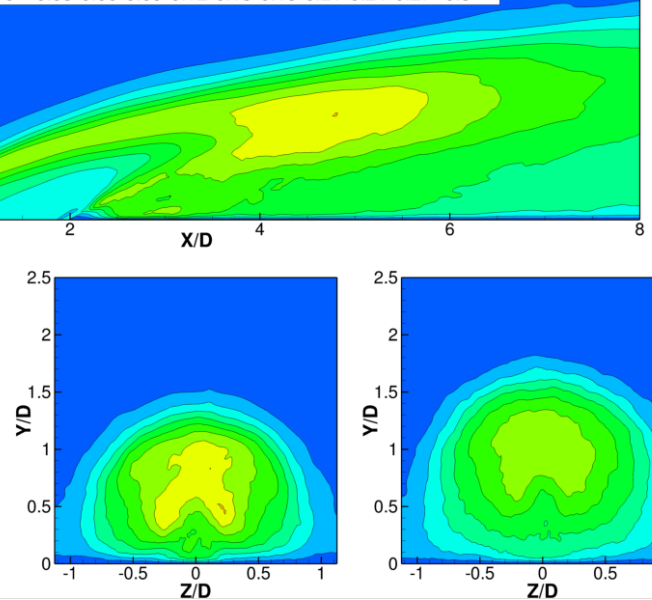

(a) Full-SEM

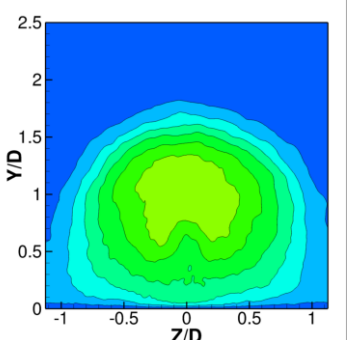

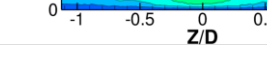
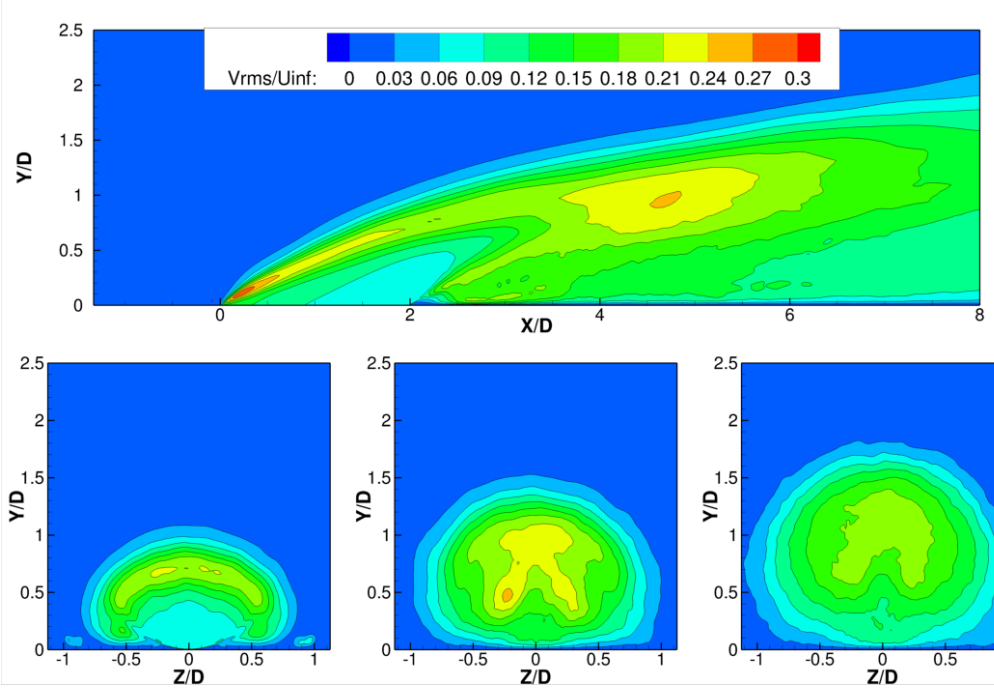

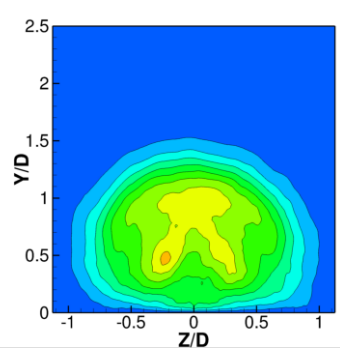

(c) Tube-SEM

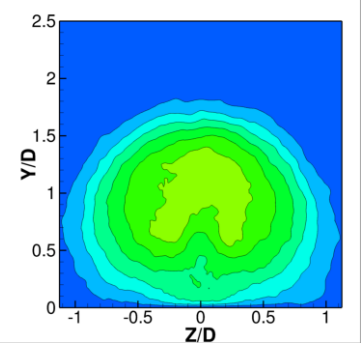

$-0.5 \underset{\text { Z/D }}{10}$

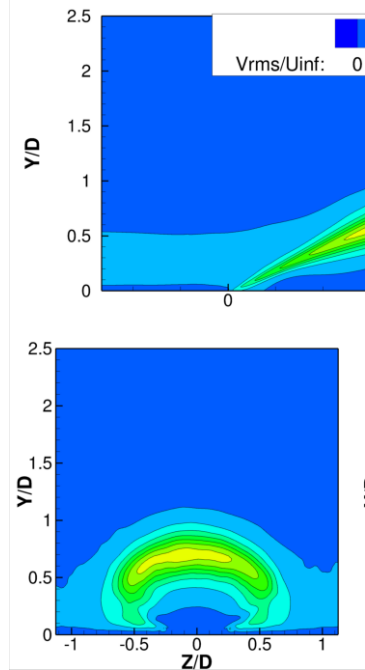

Z/D

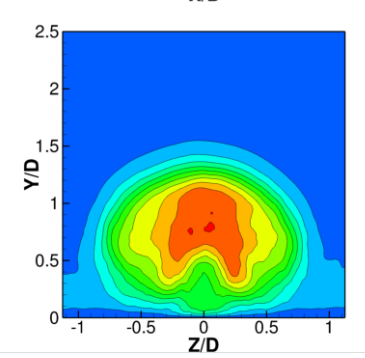

(b) Tunnel-SEM

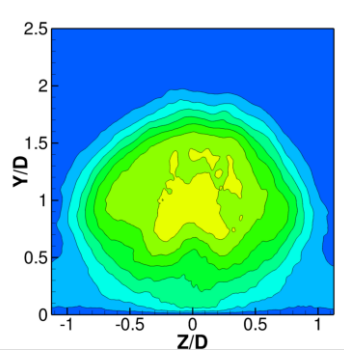

ZiD
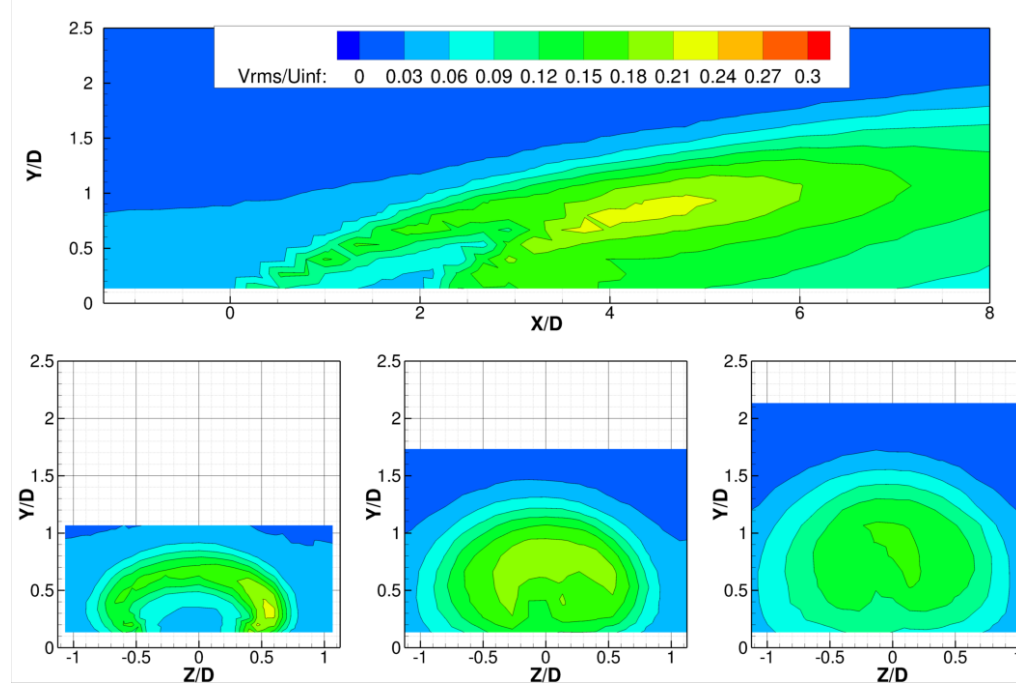

(d) U-V Probe

Figure 17. Root-mean-squared vertical velocity fluctuations contours from LES and U-V xT-wire Probe 


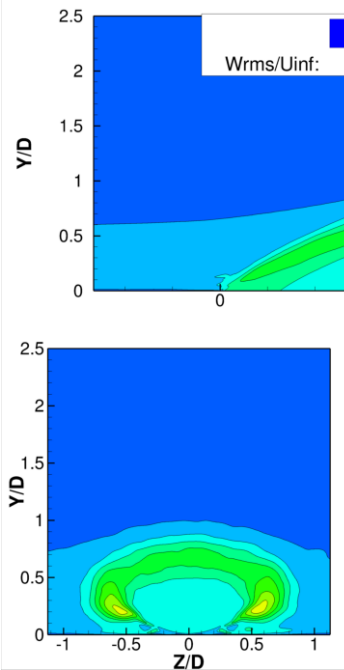

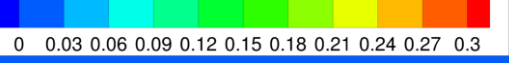
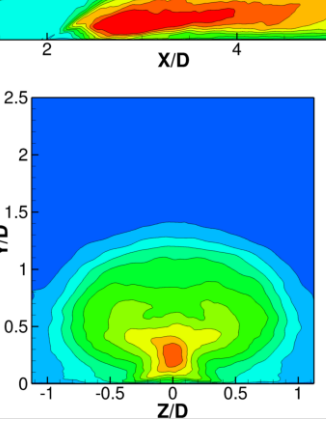

(a) Full-SEM
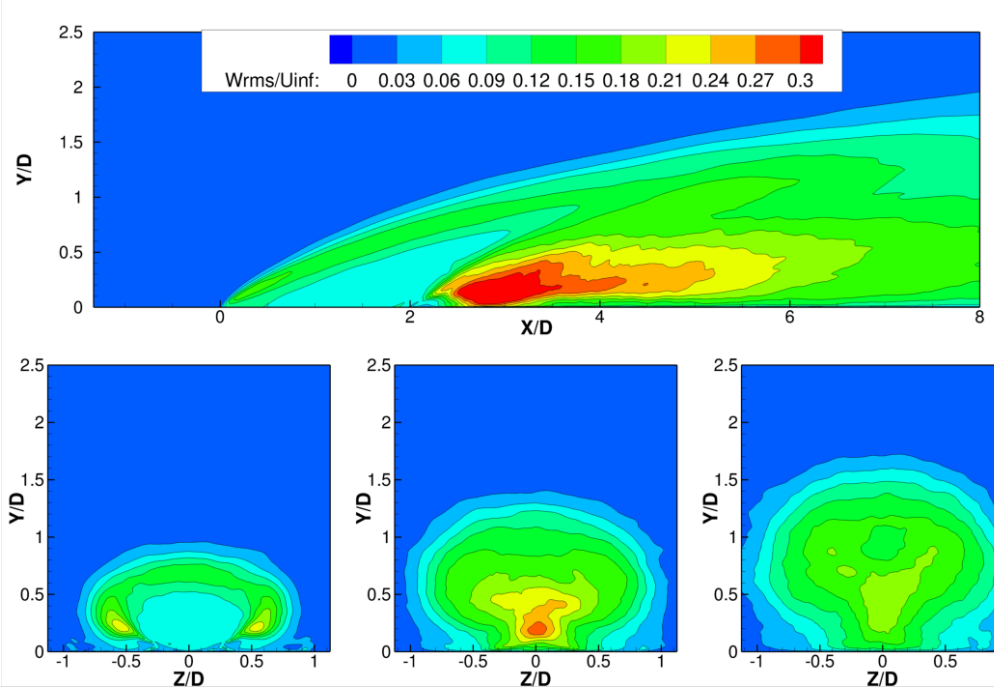

(c) Tube-SEM
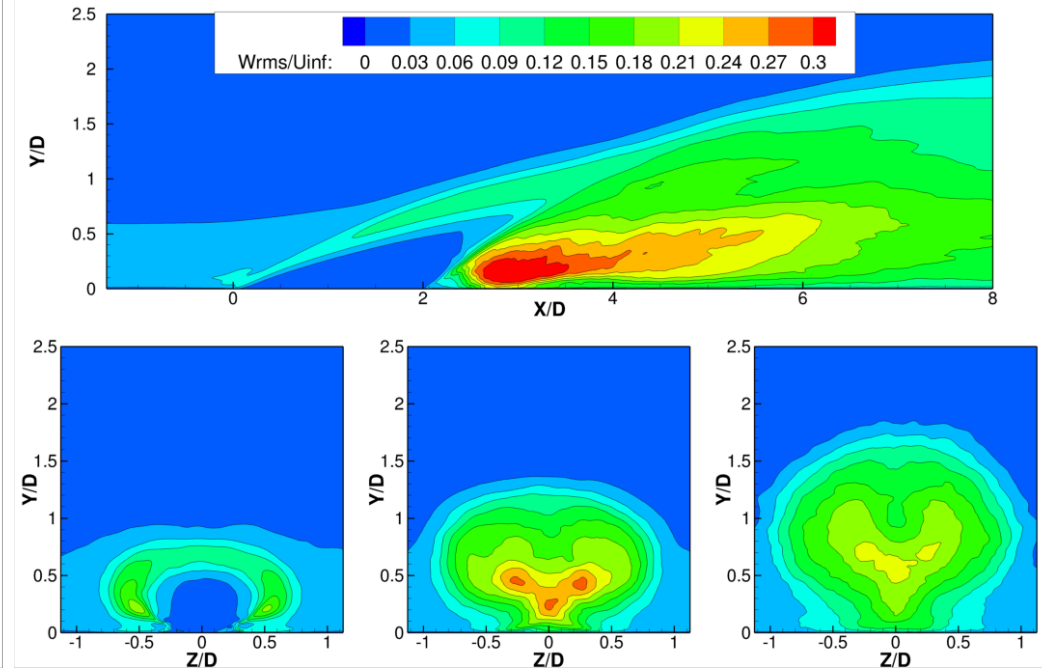

(b) Tunnel-SEM

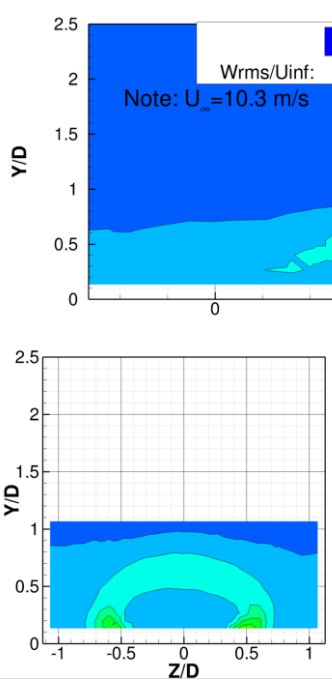

$\begin{array}{lllllllllll}0 & 0.03 & 0.06 & 0.09 & 0.12 & 0.15 & 0.18 & 0.21 & 0.24 & 0.27 & 0.3\end{array}$

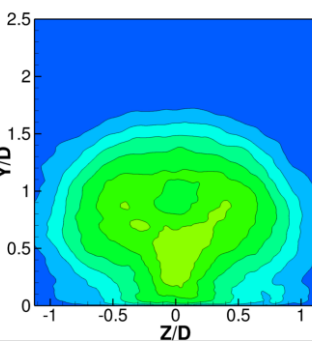

Figure 18. Root-mean-squared spanwise velocity fluctuations contours from LES and U-W xT-wire Probe 


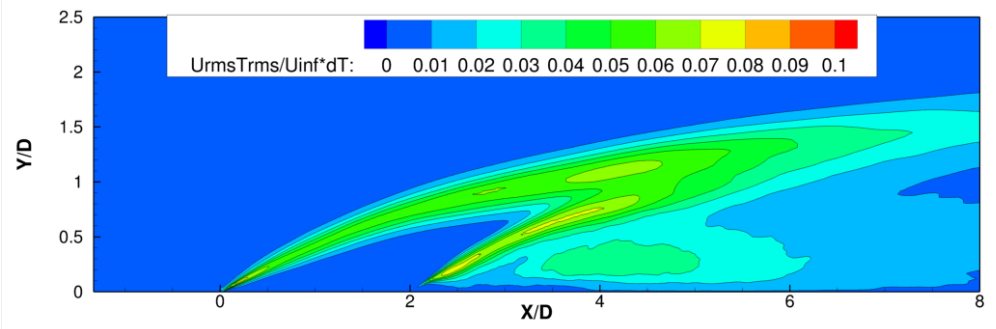

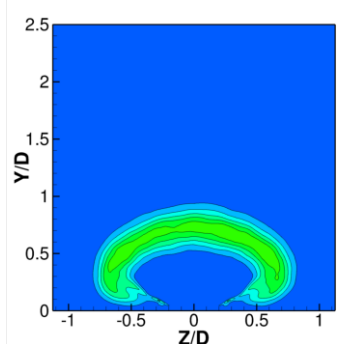

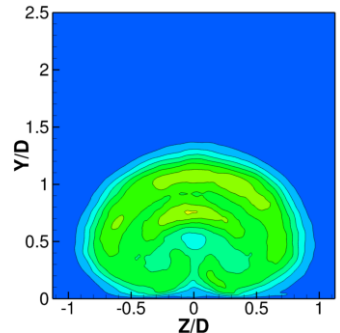

(a) Full-SEM
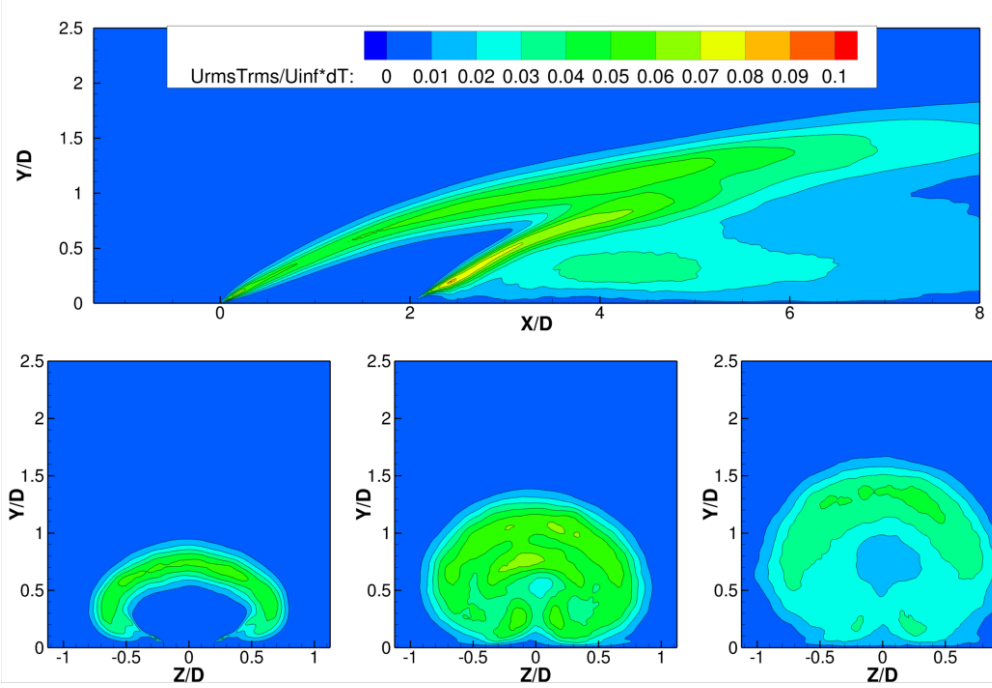
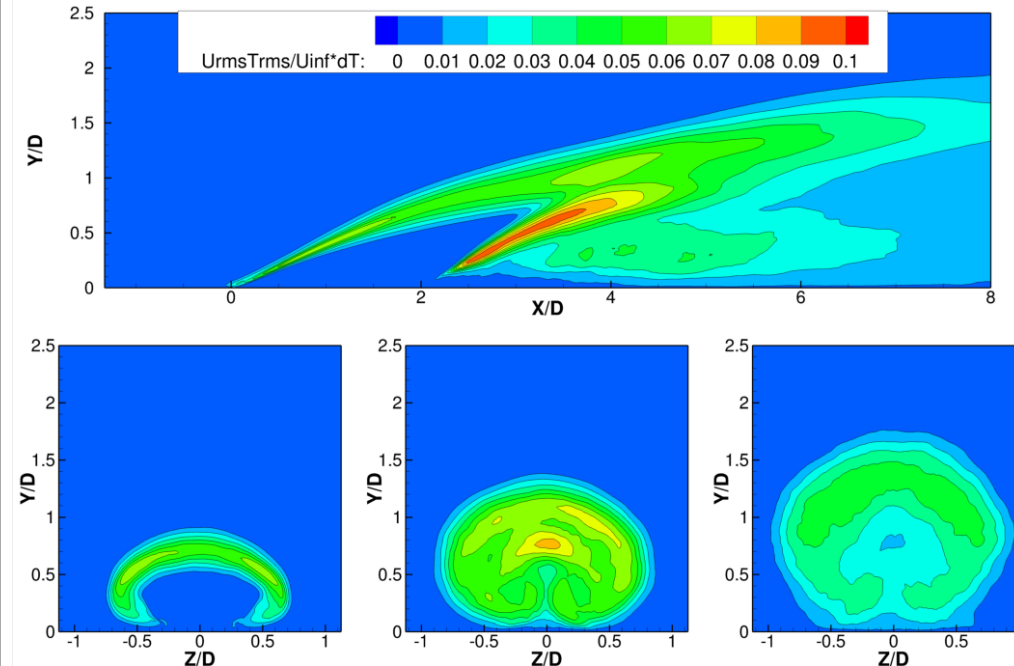

zio
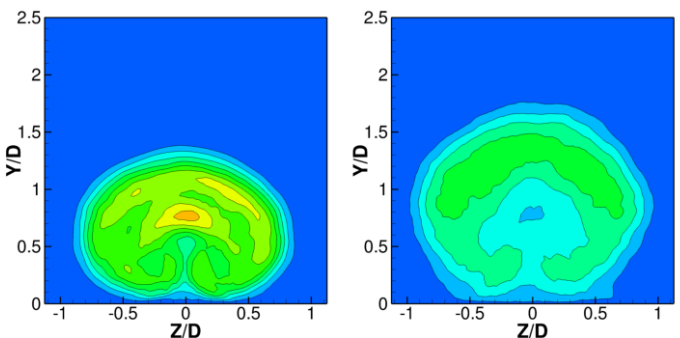

(b) Tunnel-SEM

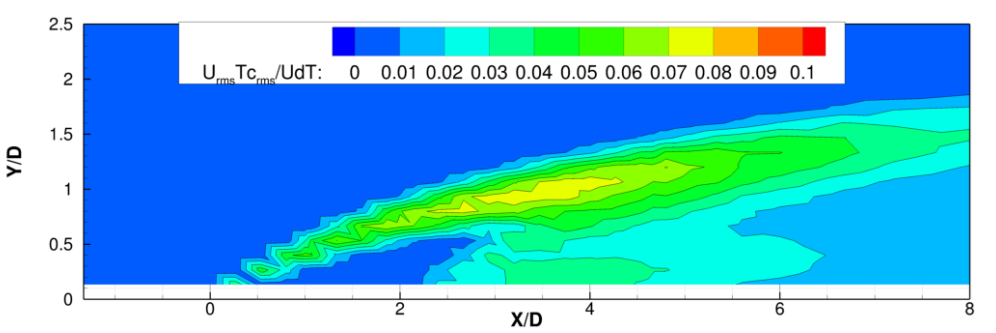

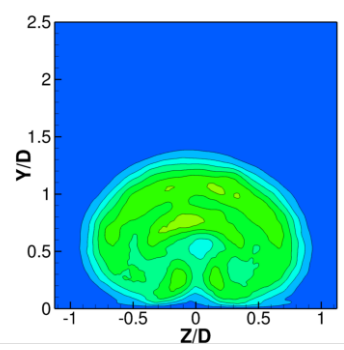

(c) Tube-SEM
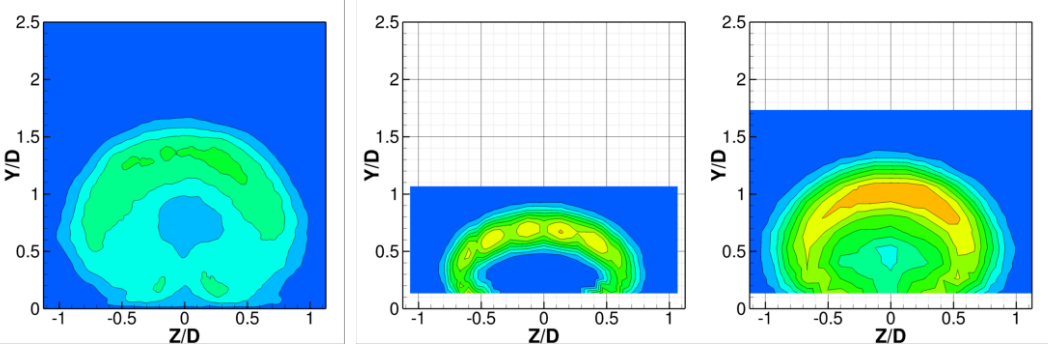

(d) U-V Probe

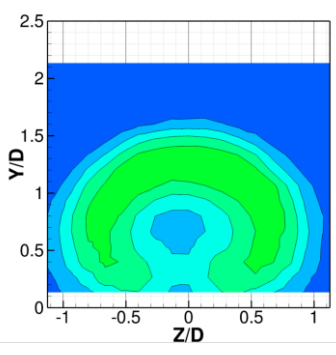

Figure 19. Root-mean-squared spanwise velocity fluctuations contours from LES and U-V xT-wire Probe 


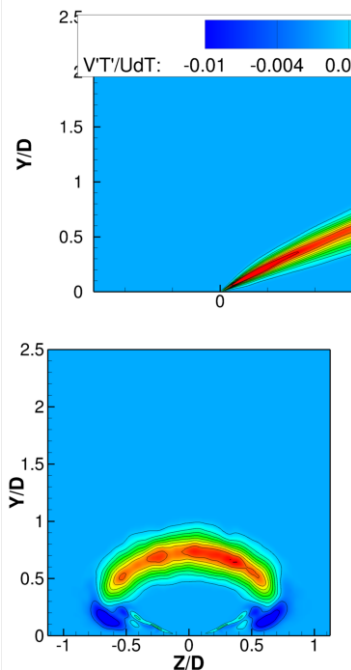

Z/D

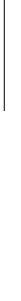
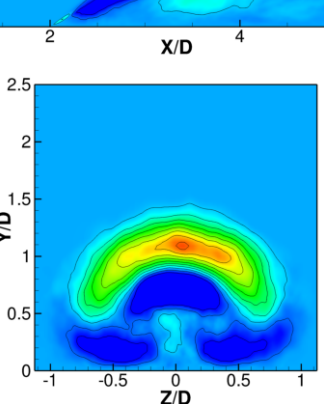

(a) Full-SEM
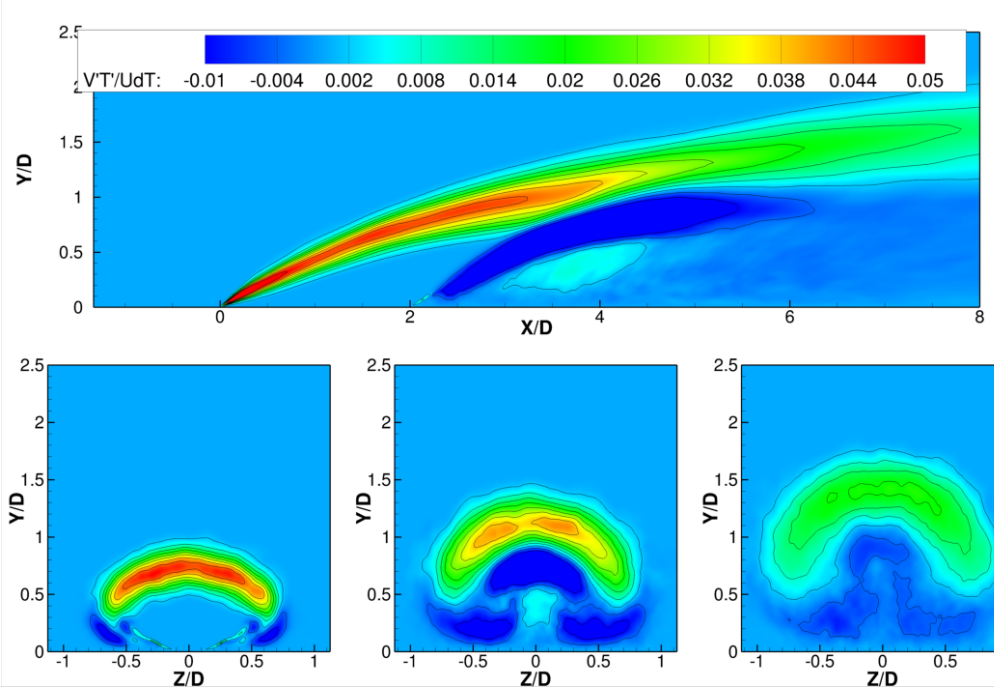

Z/D
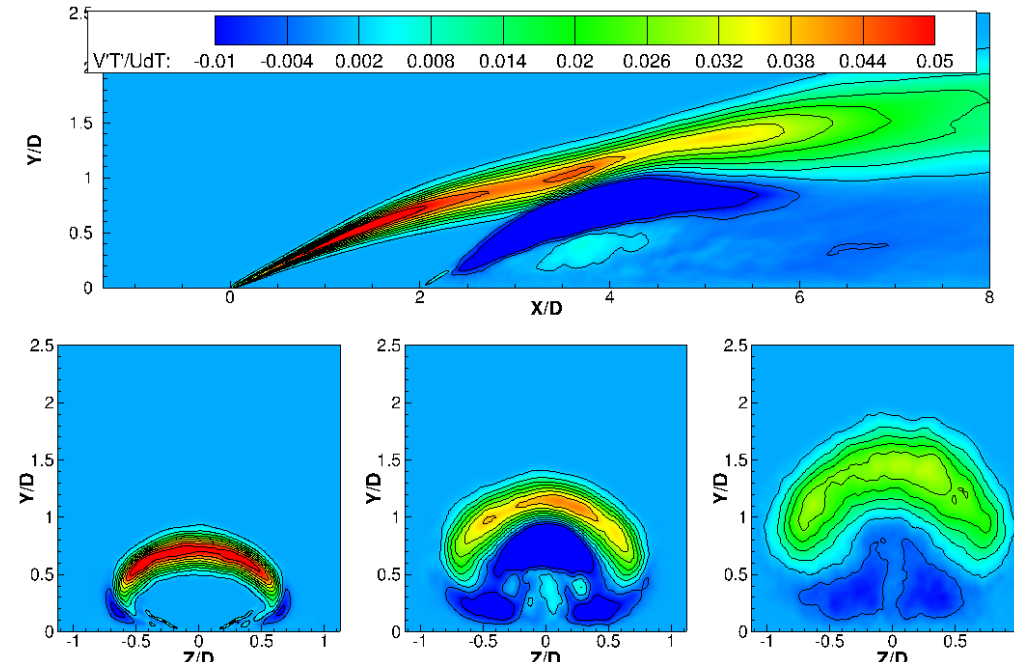

ZiD

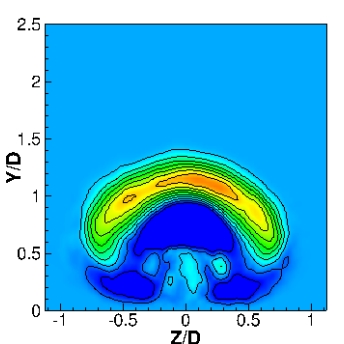

(b) Tunnel-SEM

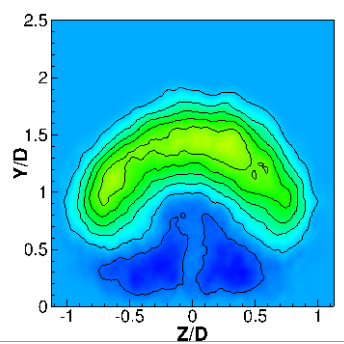

ZiD

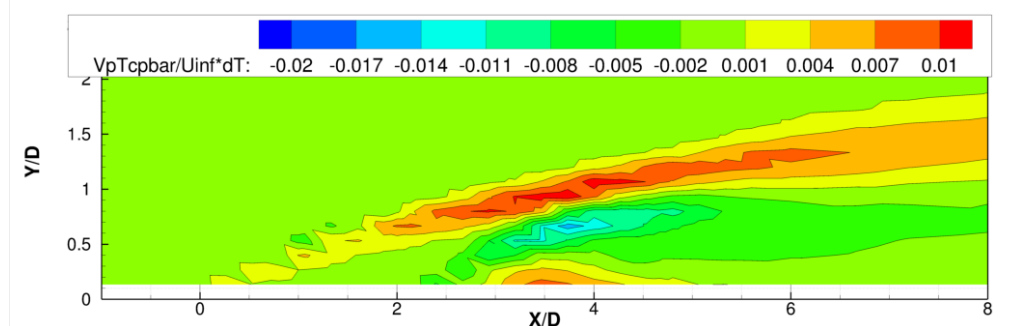

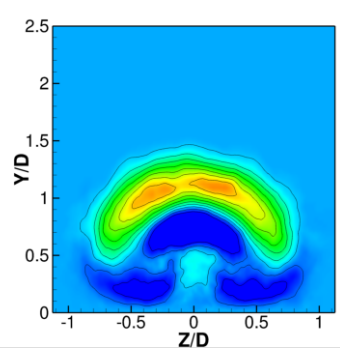

(c) Tube-SEM
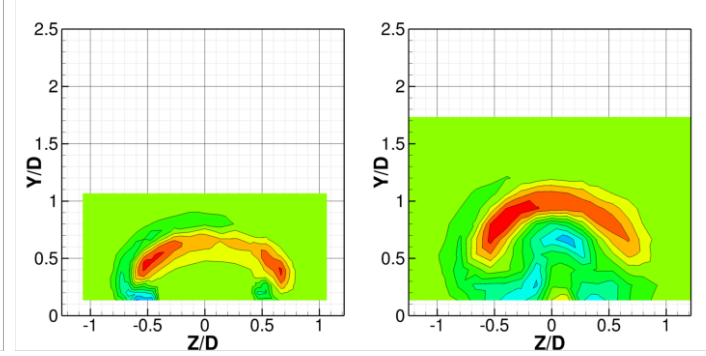

(d) U-V Probe

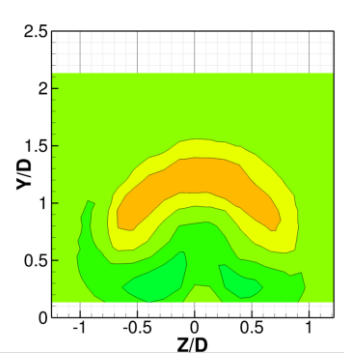

Figure 20. Streamwise turbulent heat flux $\left(\overline{V^{\prime} T^{\prime}} / U \Delta T\right)$ from LES and U-V xT-wire Probe 